\title{
29. DATA REPORT: MINERALOGIC, STRUCTURAL, AND CHEMICAL VARIABILITY OF MANTLE SECTIONS FROM HOLES 920B AND 920D ${ }^{1}$
}

\author{
Klaus-Peter Burgath, ${ }^{2}$ Vesna Marchig, ${ }^{2}$ and Kamel Mussallam ${ }^{3}$
}

\begin{abstract}
Serpentinized peridotites from selected sections from Holes 920B and 920D, drilled in the Mid-Atlantic Ridge near the Kane Transform (MARK) area, include harzburgites and minor olivine-rich lherzolites. They are characterized by porphyroclastic textures with large (up to $1.2 \mathrm{~cm}$ ), mostly ellipsoidal orthopyroxenes. The original modal composition ( $70 \%-85 \%$ olivine, $15 \%-22 \%$ orthopyroxene, $0 \%-8 \%$ clinopyroxene, and $0.5 \%-1 \%$ spinel) is close to that of refractory mantle peridotites in ophiolites and in other Mid-Atlantic Ridge regions.

The primary mineral constituents are present in a number of morphological varieties, which show evidence of high-temperature deformation and moderate recrystallization. Olivine (forsterite $[\mathrm{Fo}]=90.1-90.3 \mathrm{~mol} \%$ ) is present as kink-banded or undulatory grains up to $4 \mathrm{~mm}$ in size, and as a mosaic of equidimensional smaller grains, particularly in the vicinity of orthopyroxene porphyroclasts. Aside from porphyroclasts, orthopyroxene (wollastonite $[\mathrm{Wo}]=1.2-6.6$, enstatite $[\mathrm{En}]=84.0-89.4$, ferrosilite $[\mathrm{Fs}]=8.9-10.1 \mathrm{~mol} \%$ ) is present as small, undulatory, matrix grains, as small, anhedral, kink-banded grains in a mosaic arrangement, and as small, polygonal, undeformed grains. The porphyroclasts are heterogeneous, with more $\mathrm{Ca}, \mathrm{Al}, \mathrm{Cr}$, and $\mathrm{Ni}$ in the cores than in the rims. Small, undulatory, orthopyroxenes are chemically comparable to the rims of the porphyroclasts. The orthopyroxene in mosaic arrangement is lower in $\mathrm{Ca}, \mathrm{Ti}, \mathrm{Ni}$, and $\mathrm{Cr}$ than the porphyroclasts. We explain this by a combined process of porphyroclast disintegration and exsolution of clinopyroxene.

Clinopyroxenes display morphological varieties similar to orthopyroxene, but have a comparatively wider compositional range $(\mathrm{Wo}=40.7-50.1, \mathrm{En}=46.1-53.8$, and $\mathrm{Fs}=3.4-5.5 \mathrm{~mol} \%)$. Porphyroclastic grains display similar core-rim variations as orthopyroxenes. Undeformed interstitial clinopyroxene, believed to have formed from exsolved clinopyroxene in orthopyroxene, is chemically indistinguishable from other textural varieties. Evidence for magmatic impregnation is absent. Spinels are chromium bearing, but belong to the alumina-rich group $(\mathrm{Cr} \#=0.241-0.344)$. They are believed to have preserved their original composition during high-temperature deformation, or they may have formed as symplectites by reaction of A1-rich orthopyroxene with olivine.

The bulk chemical composition of the serpentinized peridotites was modified by alteration. Heavy rare-earth elements together with $\mathrm{Sc}, \mathrm{Al}, \mathrm{Si}$, $\mathrm{Ti}$, and $\mathrm{Cr}$ are the only elements that do not indicate mobility during alteration. All other determined elements, including light rare-earth elements, were more or less mobile. Positive europium anomalies in some samples may be due to the reduction of $\mathrm{Eu}^{3+}$ to significantly more mobile $\mathrm{Eu}^{2+}$ under conditions of low oxygen fugacity.

The platinum group elements also reveal some mobility. Enrichment of Pd in secondary sulfides is indicated. Elevated Os contents occur in samples with more chromium-bearing spinel.
\end{abstract}

\section{INTRODUCTION}

Serpentinized peridotite is exposed in a belt, $2 \mathrm{~km}$ wide and more than $20 \mathrm{~km}$ long, on the western median valley wall of the MidAtlantic Ridge at $23^{\circ} 20^{\prime} \mathrm{N}$ (Fig. 1). The peridotite outcrop was interpreted as upper mantle-derived material, which was unroofed as a consequence of extensional tectonics on the slowly spreading MidAtlantic Ridge during a period of low volcanic activity (Karson et al., 1987; Mével et al., 1991).

Two holes were drilled into peridotite in the Mid-Atlantic Ridge near the Kane Transform (MARK) area: Hole 920B penetrated 126.4 $\mathrm{m}$ below seafloor (mbsf) and Hole 920D, $16 \mathrm{~m}$ from Hole 920B, penetrated 200.8 mbsf. The cores (recovery: $37.8 \%$ in Hole $920 \mathrm{~B}, 47.4 \%$ in Hole 920D) contained serpentinized peridotites with some gabbroic intersections (Cannat et al., 1995). Thirty-four samples of serpentinized peridotite were selected for a detailed investigation. The position of these samples within the holes is shown in Figure 2.

The samples are $60 \%$ to $100 \%$ serpentinized. Relict assemblages of orthopyroxene, olivine, and the characteristic serpentine pseudo-

'Karson, J.A., Cannat, M., Miller, D.J., and Elthon, D. (Eds.), 1997. Proc. ODP, Sci. Resuits, 153: College Station, TX (Ocean Drilling Program).

${ }^{2}$ Federal Institute for Geosciences and Natural Resources, Stilliweg 2, 30655, Hannover, Federal Republic of Germany. Correspondence author, Marchig: vonrad@gatel.bgr.d400.de

'Lohhof 9, D-20535, Hamburg, Federal Republic of Germany. morphs after these phases allow estimation of the original composition (Fig. 2): olivine $70 \%-85 \%$, orthopyroxene $15 \%-22 \%$, clinopyroxene $0 \%-8 \%$, and chromium-bearing spinel $0.5 \%-1.5 \%$. The rocks can be classified as harzburgites and olivine-rich lherzolites. The modal ratios coincide with those of refractory mantle peridotites in ophiolite complexes (Coleman, 1977).

The peridotites exhibit porphyroclastic textures with large (4-12 $\mathrm{mm}$ ) orthopyroxene porphyroclasts, either as individual grains or in aggregates (Pl. 1, Fig. 1). The orthopyroxene porphyroclasts are elongated parallel or oblique to their c-axes, with angles of around $30^{\circ}$ (see Pl. 1, Fig. 1). As was stated by Nicolas and Poirier (1976), the main gliding plane is (100) and subgrains with boundaries parallel to (001) are commonly developed (Pl. 1, Fig. 1; Pl. 2, Figs. 1, 3). In many cases, the porphyroclasts are disintegrated and the subgrains display a nearly equal orientation along foliation planes. Diopside clasts with grain sizes between 3 and $4 \mathrm{~mm}$ are mainly concentrated in clusters close to large orthopyroxene porphyroclasts. Olivine exhibits smaller grain sizes $(1-2 \mathrm{~mm})$ than the pyroxenes. It is supposed that solid-state deformation of olivine has resulted in a reduction in size of originally larger grains.

Our objectives were (1) to find out the textural and chemical variations of the mantle-formed minerals (olivine, orthopyroxene, clinopyroxene, and spinel), and the chronological order of these variations, and (2) to elucidate the chemical characteristics and degree of depletion of the peridotites. 


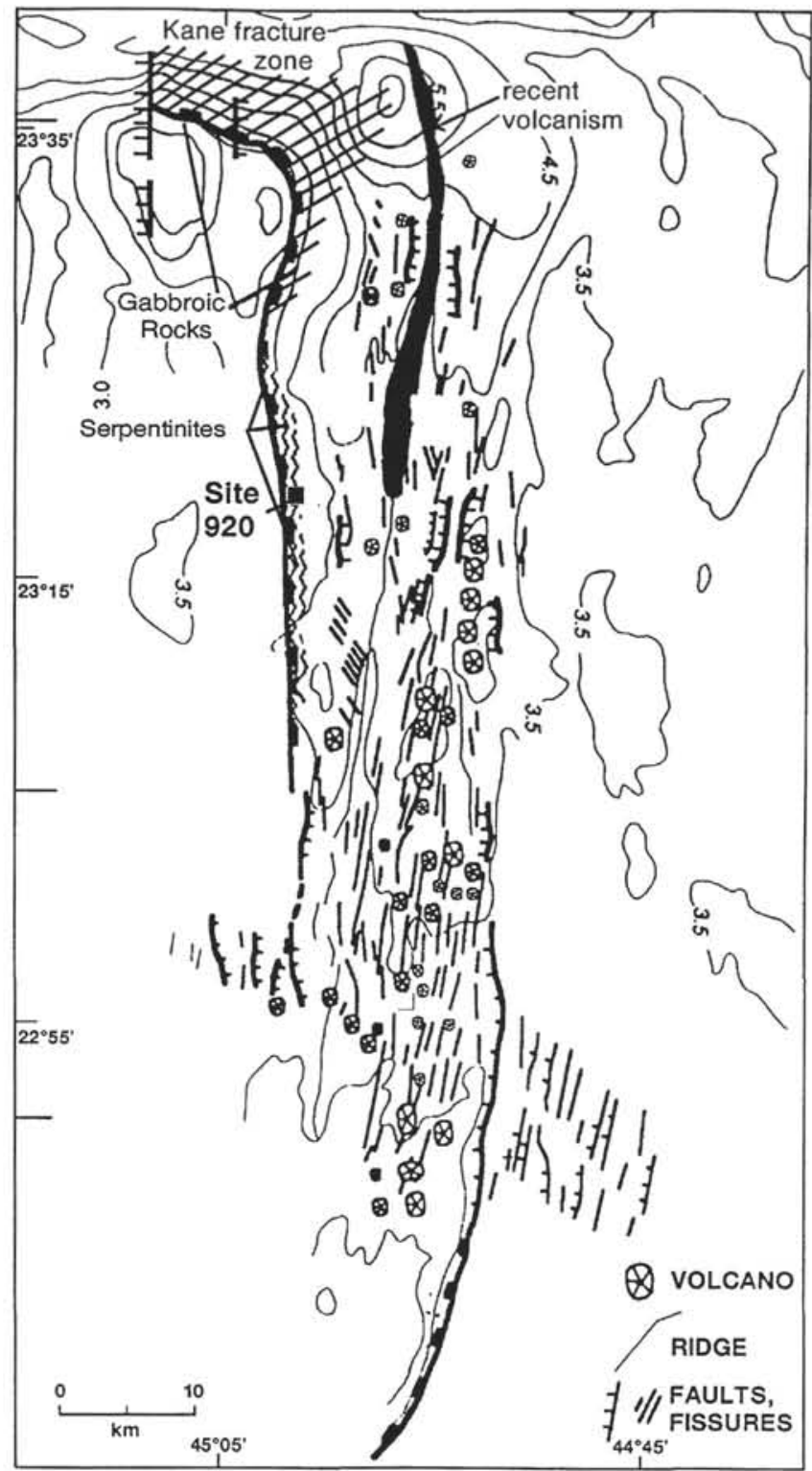

Figure 1. Sketch of tectonic setting of Site 920 on the western wall of the part of Mid-Atlantic Ridge adjacent to the Kane Fracture Zone (from Cannat et al., 1995).

\section{METHODS}

Two slices were cut from each rock sample for preparation of thin and polished sections. The sections were investigated microscopically in translucent and reflected light. Eleven thin sections were selected for microprobe analyses using a Cameca microprobe analyzer at Hamburg University. Operating conditions were $15-\mathrm{kV}$ accelerating voltage, 15-nA sample current and 10-s counting time. Natural minerals were used as standards. Entries are averages of 5-10 individual analyses in the same mineral grain.

The results of microprobe analyses on 17 olivine grains, 41 orthopyroxene grains, 54 clinopyroxene grains, and 15 spinel grains are listed in Tables 1-11.

The remaining parts of the samples were dried at $60^{\circ} \mathrm{C}$ and then crushed in a shatterbox with an agate inlay. The powdered samples were used for bulk chemical analyses. Major and trace elements were determined by X-ray fluorescence; the analyses were performed in lithium tetraborate and lithium metaborate glasses. Rare-earth ele- ments (REEs) and some trace elements below the detection limit of $\mathrm{X}$-ray fluorescence were determined using an inductively coupled plasma-mass spectrometer (ICP-MS) after dissolution under pressure in a mixture of $\mathrm{HNO}_{3}$ and $\mathrm{HF}$. Both methods were calibrated with international standards. The error is less than $2 \%$ for major elements and less than $5 \%$ for trace elements. The results of bulk chemical analyses are listed in Table 12. Analyses presented in this table, as well as those used in further statistical evaluations, were not calculated on a loss-on-ignition (LOI)-free basis.

In 14 samples of serpentinized peridotite, the contents of platinum group elements (PGEs) and gold were measured by means of instrumental neutron activation analysis (INAA) following the nickel sulfide fire assay (by Activation Laboratories Ltd., Canada). The results are listed in Table 13.

\section{RESULTS}

\section{Textural and Chemical Variations of Primary Minerals}

Olivine, orthopyroxene, clinopyroxene, and spinel are present in a number of textural and chemical varieties in the investigated harzburgites and clinopyroxene-poor lherzolites. Their characteristics are detailed below.

\section{Olivine}

Olivine is extensively replaced by serpentine. Relicts are commonly preserved in the vicinity of orthopyroxene porphyroclasts.

Three main olivine morphologies can be distinguished:

a. Large undulatory grains ("augen") with sizes up to more than $4 \mathrm{~mm}$.

b. Small undulatory grains with weak kinking (recognized at least in some grains) and with polygonal boundaries. Average size is between 1 and $2 \mathrm{~mm}$.

c. Small grains without apparent deformation. Polygonal shapes and $120^{\circ}$ triple junctions in aggregates. Average size is between 0.1 and $0.5 \mathrm{~mm}$.

The large grains are either equidimensional, with smooth and lobate surfaces, or they are elongated parallel to elongated pyroxenes (/100/ of olivine parallel to /100/ of orthopyroxene), having a lengthto-width ratio of 3.5-10 and terraced surfaces. The elongation is the result of a strong plastic strain with translation gliding under higher temperature asthenospheric conditions (Nicolas et al., 1971; Gueguen and Nicolas, 1980). Olivine inclusions in porphyroclastic orthopyroxene are commonly deformed and elongated, olivine inclusions in chromite are either round or subhedral.

\section{Chemistry}

Microprobe analyses of olivine from 9 sections from Hole 920D are listed in Table 1. This table includes analyses of large clasts, small undulatory grains, and small undeformed grains, but significant differences are not apparent.

Figure 3 shows the vertical variation of forsterite $(\mathrm{Fo})$ in large, kinked, olivine grains. Fo remains fairly constant ( 90.15 to 90.30 ), independent of the depth and the ratio of olivine to pyroxene. In olivine-spinel pairs (Table 1; analyses 8, 11, 13, 15, 16) and in an olivine included in chromite (Table 1; analysis 9 ) the Fo content increases to $>90.6$. This increase is explained by the subsolidus reequilibration between olivine and chromite and the general increase of $\mathrm{Mg}$ in olivine with increasing chromite to olivine ratio (Irvine, 1967; Burgath and Weiser, 1980; Augé,1982; Lehmann, 1983). The CaO and its variation are low $(0.01-0.04 \mathrm{wt} \%)$, and $\mathrm{NiO}$ also varies only between 0.29 and $0.36 \mathrm{wt} \%$. Similar small variations of the Fo content between 90 and 92 and $\mathrm{NiO}$ between 0.2 and 0.45 wt\% were found in olivines from ophiolitic refractory harzburgites (Augé,1982; Orberg- 

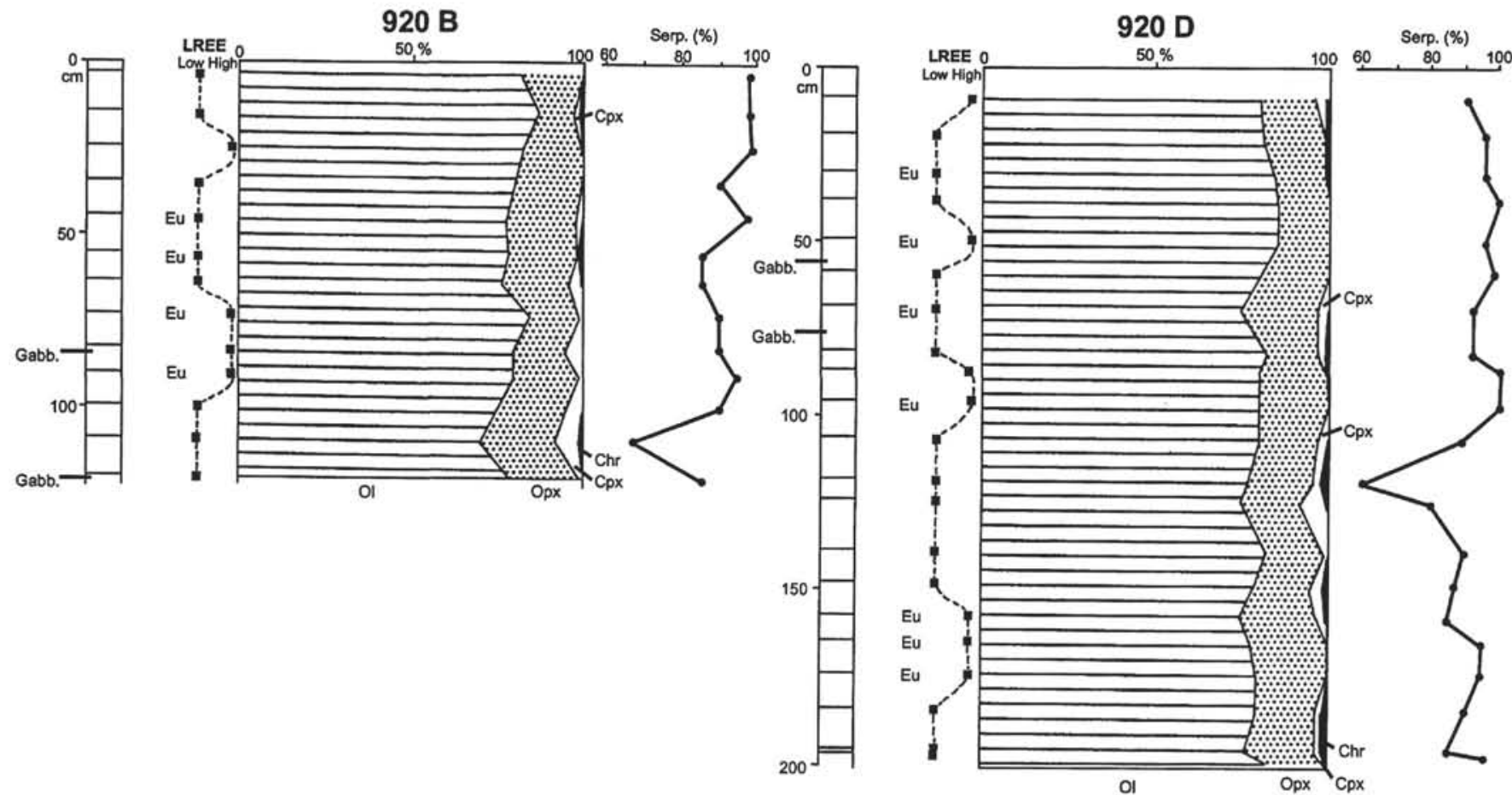

Figure 2. Modal compositions of peridotites from Holes 920B and 920D. The position of samples is marked in the column on the left side of the sketch. Also on the left are presented two types of REE patterns (low and high LREE) and the presence of a positive Eu anomaly. Percent of serpentinization is on the right. Serp. $=$ serpentine.

Table 1. Microprobe analyses of olivine, Hole 920D.

\begin{tabular}{|c|c|c|c|c|c|c|c|c|c|}
\hline $\begin{array}{l}\text { Core, section, } \\
\text { interval }(\mathrm{cm}) \text { : }\end{array}$ & $2 \mathrm{R}-1,29-36$ & $2 \mathrm{R}-1,29-36$ & $6 R-2,100-104$ & $6 \mathrm{R}-2,100-104$ & $8 R-2,62-69$ & $10 \mathrm{R}-3,8-12$ & $14 \mathrm{R}-3,68-74$ & $14 R-3,68-74$ & $14 \mathrm{R}-3,68-74$ \\
\hline $\mathrm{SiO}_{2}$ & 39.84 & 38.73 & 39.98 & 39.75 & 40.45 & 40.20 & 40.16 & 39.91 & 40.48 \\
\hline $\mathrm{TiO}_{2}^{2}$ & - & - & - & 0.01 & 0.01 & - & - & - & 0.01 \\
\hline $\mathrm{Al}_{2} \mathrm{O}_{3}$ & - & - & 0.01 & - & - & - & - & 0.01 & 0.03 \\
\hline $\mathrm{Cr}_{2} \mathrm{O}_{3}$ & - & 0.01 & 0.03 & 0.02 & - & - & 0.01 & 0.02 & 0.03 \\
\hline $\mathrm{FeO}$ & 9.74 & 9.57 & 9.65 & 9.69 & 9.67 & 9.76 & 9.62 & 9.29 & 8.55 \\
\hline $\mathrm{MnO}$ & 0.15 & 0.18 & 0.15 & 0.15 & 0.15 & 0.12 & 0.14 & 0.14 & 0.13 \\
\hline $\mathrm{MgO}$ & 50.50 & 50.32 & 50.70 & 50.14 & 50.18 & 50.18 & 49.90 & 49.73 & 49.89 \\
\hline $\mathrm{NiO}$ & 0.38 & 0.38 & 0.38 & 0.41 & 0.38 & 0.36 & 0.36 & 0.41 & 0.47 \\
\hline $\mathrm{CaO}$ & 0.01 & 0.02 & 0.04 & 0.04 & 0.03 & 0.02 & 0.04 & 0.02 & 0.04 \\
\hline Sum & 100.46 & 99.21 & 100.93 & 100.22 & 100.88 & 100.66 & 100.24 & 99.51 & 99.63 \\
\hline $\begin{array}{l}\text { Core, section, } \\
\text { interval }(\mathrm{cm}) \text { : }\end{array}$ & $15 \mathrm{R}-1,15-20$ & $15 R-1,15-20$ & $17 \mathrm{R}-4,75-81$ & $17 R-4,75-81$ & $21 R-2,74-79$ & $21 \mathrm{R}-2,74-79$ & $22 \mathrm{R}-5,37-43$ & $22 R-5,37-43$ & \\
\hline $\mathrm{SiO}_{2}$ & 39.65 & 40.70 & 39.01 & 39.94 & 39.78 & 39.60 & 39.52 & 40.49 & \\
\hline $\mathrm{TiO}_{2}$ & - & 0.01 & - & - & 0.01 & - & - & 0.01 & \\
\hline $\mathrm{Al}_{2} \mathrm{O}_{3}$ & 0.03 & 0.01 & 一 & 一 & 0.02 & - & 0.01 & 0.01 & \\
\hline $\mathrm{Cr}_{2} \mathrm{O}_{3}$ & 0.01 & 0.01 & $\overline{070}$ & - & 0.05 & - & 0.03 & - & \\
\hline $\mathrm{FeO}$ & 9.45 & 9.42 & 9.70 & 9.38 & 9.51 & 9.41 & 9.34 & 9.62 & \\
\hline $\mathrm{MnO}$ & 0.15 & 0.14 & 0.15 & 0.13 & 0.15 & 0.14 & 0.15 & 0.25 & \\
\hline $\mathrm{MgO}$ & 49.73 & 50.28 & 49.89 & 50.62 & 50.30 & 49.93 & 50.18 & 49.96 & \\
\hline $\mathrm{NiO}$ & 0.37 & 0.36 & 0.39 & 0.40 & 0.41 & 0.42 & 0.40 & 0.42 & \\
\hline $\mathrm{CaO}$ & 0.02 & 0.03 & 0.03 & 0.04 & 0.03 & 0.02 & 0.04 & 0.02 & \\
\hline Sum & 99.41 & 100.95 & 99.19 & 100.53 & 100.26 & 99.52 & 99.68 & 100.77 & \\
\hline
\end{tabular}

Note: $-=$ not detected.

er et al., 1995) and in peridotites from other oceanic regions (Hamlyn and Bonatti, 1981; Dick et al., 1984).

\section{Orthopyroxene}

Five morphological varieties of orthopyroxene can be distinguished:

a. Rounded or ellipsoidal porphyroclasts with grain sizes ranging from 4 to $12 \mathrm{~mm}$ and aspect ratios from 1.5 to 3 , with exsolutions of clinopyroxene parallel to (100) of orthopyroxene. (Pl. 1, Figs. 1, 4; Pl. 2, Figs. 1, 3).

b. Individual small grains with sizes between 0.5 and $2 \mathrm{~mm}$. Frequent intergrowth with vermiform or amoeboidal spinel, and with thin clinopyroxene exsolution lamellae (Pl. 1, Fig. 2).

c. Mosaic aggregates of anhedral grains with sizes between 0.3 and $1 \mathrm{~mm}$, and with similar optical orientation (subparallel $\mathrm{Z}$ axes) (PI. 1, Fig. 3). Occasional intergrowth with amoeboidal spinel. 
Table 2. Microprobe analyses of orthopyroxene porphyroclasts, Hole 920D.

\begin{tabular}{|c|c|c|c|c|c|c|c|c|c|}
\hline $\begin{array}{l}\text { Core, section, } \\
\text { interval }(\mathrm{cm}) \text { : }\end{array}$ & $2 \mathrm{R}-1,29-36$ & $2 \mathrm{R}-1,29-36$ & $6 \mathrm{R}-2,100-104$ & $6 \mathrm{R}-2,100-104$ & $6 \mathrm{R}-2,62-69$ & $8 R-2,62-69$ & $8 \mathrm{R}-2,8-12$ & $10 \mathrm{R}-3,8-12$ & $10 \mathrm{R}-3,8-12$ \\
\hline $\mathrm{SiO}_{2}$ & 53.480 & 54.160 & 53.498 & 53.461 & 54.710 & 54.580 & 54.310 & 54.550 & 54.850 \\
\hline $\mathrm{TiO}_{2}^{2}$ & 0.050 & 0.039 & 0.093 & 0.046 & 0.035 & 0.028 & 0.054 & 0.040 & 0.046 \\
\hline $\mathrm{Al}_{2} \mathrm{O}_{3}$ & 4.375 & 4.155 & 4.898 & 4.145 & 3.575 & 3.636 & 3.871 & 3.497 & 3.579 \\
\hline $\mathrm{Cr}_{2} \mathrm{O}_{3}$ & 0.986 & 0.907 & 1.066 & 0.868 & 0.740 & 0.701 & 1.009 & 0.720 & 0.682 \\
\hline $\mathrm{FeO}$ & 6.553 & 6.233 & 6.013 & 5.968 & 6.266 & 6.286 & 6.000 & 6.365 & 6.050 \\
\hline $\mathrm{MnO}$ & 0.14 & 0.139 & 0.106 & 0.118 & 0.140 & 0.160 & 0.107 & 0.117 & 0.137 \\
\hline $\mathrm{MgO}$ & 32.88 & 33.616 & 32.619 & 31.941 & 33.840 & 34.020 & 33.255 & 32.950 & 33.110 \\
\hline $\mathrm{CaO}$ & 0.919 & 1.204 & 2.823 & 3.492 & 1.420 & 0.984 & 2.330 & 1.501 & 2.027 \\
\hline $\mathrm{NiO}$ & 0.092 & 0.096 & 0.112 & 0.084 & 0.119 & 0.049 & 0.092 & 0.040 & 0.088 \\
\hline $\mathrm{Na}_{2} \mathrm{O}$ & 0.003 & 0.003 & 0.032 & 0.027 & 0.003 & 0.003 & 0.016 & 0.015 & 0.007 \\
\hline $\mathrm{K}_{2} \mathrm{O}$ & 0.001 & 0.001 & 0.001 & 0.001 & 0.001 & 0.007 & 0.001 & 0.001 & 0.001 \\
\hline Sum & 99.48 & 100.56 & 101.26 & 100.15 & 100.85 & 100.45 & 101.05 & 99.80 & 100.58 \\
\hline
\end{tabular}

\begin{tabular}{|c|c|c|c|c|c|c|c|c|}
\hline $\begin{array}{l}\text { Core, section, } \\
\text { interval }(\mathrm{cm}) \text { : }\end{array}$ & $14 \mathrm{R}-3,68-74$ & $15 \mathrm{R}-1,15-20$ & $17 R-4,75-81$ & $18 \mathrm{R}-4,15-20$ & $21 \mathrm{R}-2,74-79$ & $21 \mathrm{R}-2,74-79$ & $22 \mathrm{R}-5,37-43$ & $22 \mathrm{R}-5,37-43$ \\
\hline $\mathrm{SiO}_{2}$ & 54.290 & 54.390 & 54.975 & 55.267 & 53.158 & 53.650 & 53.720 & 53.870 \\
\hline $\mathrm{TiO}_{2}$ & 0.045 & 0.038 & 0.033 & 0.053 & 0.044 & 0.042 & 0.027 & 0.045 \\
\hline $\mathrm{Al}_{2} \mathrm{O}_{3}$ & 3.964 & 3.758 & 3.843 & 3.957 & 3.602 & 3.470 & 4.451 & 4.204 \\
\hline $\mathrm{Cr}_{2} \mathrm{O}_{3}$ & 0.853 & 0.771 & 0.775 & 0.888 & 0.779 & 0.775 & 0.969 & 0.920 \\
\hline $\mathrm{FeO}$ & 6.115 & 6.268 & 6.155 & 6.309 & 6.037 & 6.070 & 6.170 & 6.158 \\
\hline $\mathrm{MnO}$ & 0.122 & 0.130 & 0.120 & 0.176 & 0.159 & 0.180 & 0.125 & 0.144 \\
\hline $\mathrm{MgO}$ & 33.289 & 32.576 & 33.870 & 32.377 & 32.635 & 33.440 & 33.020 & 32.970 \\
\hline $\mathrm{CaO}$ & 1.460 & 1.560 & 1.106 & 1.440 & 2.725 & 1.626 & 1.499 & 2.089 \\
\hline $\mathrm{NiO}$ & 0.110 & 0.101 & 0.104 & 0.093 & 0.080 & 0.112 & 0.119 & 0.109 \\
\hline $\mathrm{Na}_{2} \mathrm{O}$ & 0.009 & 0.003 & 0.003 & 0.003 & 0.003 & 0.003 & 0.012 & 0.003 \\
\hline $\mathrm{K}_{2} \mathrm{O}$ & 0.007 & 0.001 & 0.005 & 0.001 & 0.001 & 0.001 & 0.001 & 0.001 \\
\hline Sum & 100.26 & 99.60 & 100.99 & 100.56 & 99.22 & 99.37 & 100.12 & 100.51 \\
\hline
\end{tabular}

Table 3. Microprobe profiles in two orthopyroxene porphyroclasts, Hole 920D.

Core, section,

interval (cm): 6R-2, 100-104 6R-2, 100-104 6R-2, 100-104 6R-2, 100-104 6R-2, 100-104 6R-2, 100-104 15R-1, 15-20 15R-1, 15-20 15R-1, 15-20 15R-1, 15-20

\begin{tabular}{|c|c|c|c|c|c|c|c|c|c|c|}
\hline $\mathrm{SiO}_{2}$ & 53.37 & 54.27 & 53.59 & 53.49 & 53.50 & 53.46 & 54.42 & 55.03 & 54.72 & 54.89 \\
\hline $\mathrm{TiO}_{2}^{2}$ & 0.05 & 0.07 & 0.10 & 0.06 & 0.09 & 0.05 & 0.02 & 0.03 & 0.03 & 0.04 \\
\hline $\mathrm{Al}_{2} \mathrm{O}_{3}$ & 4.83 & 4.98 & 5.30 & 5.44 & 4.90 & 4.14 & 3.57 & 4.05 & 4.00 & 3.90 \\
\hline $\mathrm{Cr}_{2} \mathrm{O}_{3}$ & 1.03 & 1.13 & 1.17 & 1.04 & 1.07 & 0.87 & 0.73 & 0.85 & 0.84 & 0.78 \\
\hline $\mathrm{FeO}$ & 6.17 & 6.18 & 5.92 & 6.34 & 6.01 & 5.97 & 6.20 & 6.03 & 5.96 & 6.09 \\
\hline $\mathrm{MnO}$ & 0.15 & 0.09 & 0.14 & 0.16 & 0.11 & 0.12 & 0.20 & 0.13 & 0.14 & 0.10 \\
\hline $\mathrm{MgO}$ & 32.26 & 31.92 & 30.89 & 33.49 & 32.62 & 31.94 & 32.24 & 32.72 & 31.94 & 32.35 \\
\hline $\mathrm{CaO}$ & 0.88 & 2.22 & 3.48 & 0.75 & 2.82 & 3.49 & 1.50 & 2.22 & 2.39 & 1.60 \\
\hline $\mathrm{NiO}$ & 0.07 & 0.11 & 0.14 & 0.12 & 0.11 & 0.08 & 0.08 & 0.13 & 0.12 & 0.08 \\
\hline $\mathrm{Na}_{2} \mathrm{O}$ & 0.00 & 0.01 & 0.01 & 0.00 & 0.03 & 0.03 & 0.01 & 0.00 & 0.01 & 0.00 \\
\hline $\mathrm{K}_{2} \mathrm{O}$ & 0.00 & 0.01 & 0.00 & 0.00 & 0.00 & 0.00 & 0.01 & 0.00 & 0.00 & 0.00 \\
\hline Süm & 98.81 & 100.99 & 100.74 & 100.89 & 101.26 & 100.15 & 98.98 & 101.19 & 100.15 & 99.83 \\
\hline
\end{tabular}

Table 4. Microprobe analyses of small deformed orthopyroxenes (1-3) and orthopyroxenes in mosaic aggregates (4-9), Hole 920D.

\begin{tabular}{|c|c|c|c|c|c|c|c|c|c|}
\hline $\begin{array}{l}\text { Core, section, } \\
\text { interval }(\mathrm{cm}) \text { : }\end{array}$ & $13 \mathrm{R}-3,21-26$ & $21 \mathrm{R}-2,74-79$ & $21 \mathrm{R}-2,74-79$ & $22 \mathrm{R}-5,37-43$ & $22 \mathrm{R}-5,37-43$ & $18 \mathrm{R}-4,15-20$ & $18 \mathrm{R}-4,15-20$ & $18 \mathrm{R}-4,15-20$ & $22 \mathrm{R}-5,37-43$ \\
\hline $\mathrm{SiO}_{2}$ & 54.34 & 54.82 & 54.33 & 55.53 & 55.36 & 54.96 & 54.81 & 54.77 & 54.07 \\
\hline $\mathrm{TiO}_{2}^{2}$ & 0.04 & 0.04 & 0.03 & 0.04 & 0.02 & 0.05 & 0.04 & 0.05 & 0.04 \\
\hline $\mathrm{Al}_{2} \mathrm{O}_{3}$ & 4.10 & 3.91 & 3.74 & 3.58 & 3.25 & 4.12 & 4.29 & 4.02 & 3.88 \\
\hline $\mathrm{Cr}_{2} \mathrm{O}_{3}$ & 0.90 & 0.76 & 0.82 & 0.60 & 0.48 & 0.94 & 0.95 & 0.90 & 0.82 \\
\hline $\mathrm{FeO}$ & 6.09 & 6.09 & 5.97 & 6.25 & 6.24 & 6.12 & 6.08 & 6.16 & 6.16 \\
\hline $\mathrm{MnO}$ & 0.16 & 0.12 & 0.15 & 0.12 & 0.16 & 0.15 & 0.14 & 0.14 & 0.14 \\
\hline $\mathrm{MgO}$ & 31.83 & 32.49 & 32.17 & 33.70 & 34.06 & 33.42 & 33.07 & 32.69 & 33.35 \\
\hline $\mathrm{CaO}$ & 2.31 & 1.32 & 1.82 & 0.95 & 0.63 & 1.45 & 1.45 & 1.59 & 1.50 \\
\hline $\mathrm{NiO}$ & 0.09 & - & - & - & - & - & - & - & 0.08 \\
\hline $\mathrm{Na}_{2} \mathrm{O}$ & 0.02 & 0.00 & 0.01 & 0.01 & 0.00 & 0.00 & 0.00 & 0.00 & 0.00 \\
\hline $\mathrm{K}_{2} \mathrm{O}$ & 0.01 & 0.00 & 0.01 & 0.00 & 0.00 & 0.01 & 0.01 & 0.00 & 0.00 \\
\hline Sum & 99.87 & 99.55 & 99.05 & 100.78 & 100.20 & 100.26 & 100.84 & 100.32 & 100.03 \\
\hline
\end{tabular}

Note: $-=$ not detected.

d. Aggregates of polygonal grains (sizes $0.1-0.3 \mathrm{~mm}$ ) with triple junctions, often together with recrystallized olivine, along surfaces and between elongated porphyroclasts (Pl. 2, Fig. 3).

e. Polygonal grains $(0.2 \mathrm{~mm}$ in size $)$ at the contact between deformed clinopyroxene grains. Connected with orthopyroxene exsolution lamellae in clinopyroxene (Pl. 2, Fig. 2).

Most grains of the varieties a and b are kinked subparallel to (001) crystallographic axes (PI. 1, Fig.1; Pl. 2, Fig. 3). Elongated porphyroclasts define a foliation plane (sub)parallel to (100) of orthopyrox- ene. These textures are typical of solid-state flow deformation in the mantle (Gueguen and Nicolas, 1980).

The individual small grains show the same deformational features as the porphyroclasts, but kink banding is better expressed. These orthopyroxenes are often associated with spinel in "myrmekitic" intergrowths (interdigitation of vermiform spinel and massive orthopyroxene; Pl. 1, Fig. 2). The orthopyroxene porphyroclasts and the matrix grains are both characterized by lobate surfaces.

The mosaic aggregates are composed of angular grains with gentle embayments and interstitial clinopyroxene; $120^{\circ}$ triple junctions of the orthopyroxene grains are common. The orientation of these or- 
Table 5. Microprobe analyses of undeformed orthopyroxene, Hole 920D.

\begin{tabular}{|c|c|c|c|c|c|}
\hline $\begin{array}{l}\text { Core, section, } \\
\text { interval }(\mathrm{cm}) \text { : }\end{array}$ & $13 R-3,21-26$ & $21 \mathrm{R}-2,74-79$ & $21 \mathrm{R}-2,74-79$ & $21 \mathrm{R}-2,74-79$ & $21 \mathrm{R}-2,74-79$ \\
\hline $\mathrm{SiO}_{2}$ & 54.93 & 54.74 & 54.82 & 55.37 & 53.43 \\
\hline $\mathrm{TiO}_{2}^{2}$ & 0.02 & 0.02 & 0.03 & 0.02 & 0.03 \\
\hline $\mathrm{Al}_{2} \mathrm{O}_{3}$ & 3.94 & 4.31 & 4.29 & 3.98 & 5.90 \\
\hline $\mathrm{Cr}_{2} \mathrm{O}_{3}$ & 0.83 & 0.94 & 0.90 & 0.90 & 1.19 \\
\hline $\mathrm{FeO}^{3}$ & 6.50 & 6.62 & 6.52 & 6.46 & 7.17 \\
\hline $\mathrm{MnO}$ & 0.15 & 0.15 & 0.16 & 0.12 & 0.19 \\
\hline $\mathrm{MgO}$ & 33.36 & 32.86 & 33.07 & 33.51 & 31.92 \\
\hline $\mathrm{CaO}$ & 0.92 & 1.21 & 0.96 & 0.86 & 0.84 \\
\hline $\mathrm{NiO}$ & 0.08 & - & - & & \\
\hline $\mathrm{Na}_{2} \mathrm{O}$ & 0.00 & $\overline{0.01}$ & 0.00 & 0.00 & 0.00 \\
\hline $\mathrm{K}_{2} \mathrm{O}$ & 0.00 & 0.00 & 0.00 & 0.00 & 0.01 \\
\hline Sum & 100.73 & 100.86 & 100.74 & 101.24 & 100.68 \\
\hline
\end{tabular}

Note: Analyses 1-4 are from polygonal grains, analysis 5 is of exsolution from clinopyroxene, $-=$ not detected.

Table 6. Microprobe analyses of deformed clinopyroxene, Hole 920D.

Core, section,

interval (cm): $\quad 8 R-2,62-69 \quad 14 R-3,68-74 \quad 15 R-1,15-20 \quad 15 R-1,15-20 \quad 15 R-1,15-20 \quad 15 R-1,15-20 \quad 15 R-1,15-20 \quad 15 R-1,15-20 \quad 15 R-1,15-20$

\begin{tabular}{|c|c|c|c|c|c|c|c|c|c|}
\hline $\mathrm{SiO}_{2}$ & 50.440 & 50.450 & 50.580 & 50.570 & 51.090 & 52.170 & 50.220 & 49.300 & 50.190 \\
\hline $\mathrm{TiO}_{2}^{2}$ & 0.104 & 0.109 & 0.087 & 0.090 & 0.078 & 0.083 & 0.099 & 0.088 & 0.096 \\
\hline $\mathrm{Al}_{2} \mathrm{O}_{3}$ & 5.118 & 5.655 & 5.362 & 5.396 & 5.171 & 4.293 & 4.777 & 4.779 & 4.869 \\
\hline $\mathrm{Cr}_{2} \mathrm{O}_{3}$ & 1.374 & 1.380 & 1.243 & 1.282 & 1.383 & 0.953 & 1.311 & 1.300 & 1.149 \\
\hline $\mathrm{FeO}$ & 2.630 & 3.090 & 3.130 & 3.270 & 3.050 & 3.270 & 2.770 & 2.580 & 2.800 \\
\hline $\mathrm{MnO}$ & 0.106 & 0.091 & 0.110 & 0.086 & 0.100 & 0.110 & 0.080 & 0.100 & 0.120 \\
\hline $\mathrm{MgO}$ & 17.240 & 17.070 & 17.870 & 17.990 & 17.370 & 18.870 & 18.450 & 17.640 & 17.790 \\
\hline $\mathrm{CaO}$ & 22.471 & 21.930 & 21.280 & 21.110 & 22.340 & 20.940 & 20.900 & 22.750 & 22.400 \\
\hline $\mathrm{NiO}$ & 0.033 & 0.062 & 0.068 & 0.068 & 0.063 & 0.046 & 0.097 & 0.070 & 0.066 \\
\hline $\mathrm{Na}_{2} \mathrm{O}$ & 0.018 & 0.052 & 0.037 & 0.051 & 0.050 & 0.062 & 0.063 & 0.053 & 0.052 \\
\hline $\mathrm{K}_{2} \mathrm{O}$ & 0.001 & 0.001 & 0.006 & 0.001 & 0.001 & 0.001 & 0.001 & 0.003 & 0.002 \\
\hline Sum & 99.62 & 99.79 & 99.76 & 99.91 & 100.69 & 100.80 & 98.77 & 98.66 & 99.53 \\
\hline
\end{tabular}

Core, section,

interval (cm): $\quad 17 \mathrm{R}-4,75-81 \quad 17 \mathrm{R}-4,75-81 \quad 18 \mathrm{R}-4,15-20 \quad 18 \mathrm{R}-4,15-20 \quad 18 \mathrm{R}-4,15-20 \quad 21 \mathrm{R}-2,74-79 \quad 21 \mathrm{R}-2,74-79 \quad 22 \mathrm{R}-5,74-79 \quad 22 \mathrm{R}-5,37-43$

\begin{tabular}{|c|c|c|c|c|c|c|c|c|c|}
\hline $\mathrm{SiO}_{2}$ & 51.810 & 49.890 & 51.290 & 49.370 & 51.090 & 49.610 & 49.790 & 50.720 & 51.440 \\
\hline $\mathrm{TiO}_{2}$ & 0.078 & 0.105 & 0.115 & 0.094 & 0.135 & 0.102 & 0.094 & 0.112 & 0.090 \\
\hline $\mathrm{Al}_{2} \mathrm{O}_{3}$ & 3.241 & 4.668 & 5.208 & 4.968 & 5.148 & 4.981 & 5.367 & 4.608 & 4.469 \\
\hline $\mathrm{Cr}_{2} \mathrm{O}_{3}$ & 0.758 & 1.223 & 1.435 & 1.314 & 1.443 & 1.283 & 1.363 & 1.176 & 1.073 \\
\hline $\mathrm{FeO}$ & 2.130 & 2.494 & 2.500 & 3.200 & 2.440 & 2.310 & 2.590 & 2.630 & 2.770 \\
\hline $\mathrm{MnO}$ & 0.080 & 0.100 & 0.086 & 0.128 & 0.050 & 0.100 & 0.110 & 0.100 & 0.090 \\
\hline $\mathrm{MgO}$ & 17.650 & 17.280 & 16.450 & 19.950 & 16.630 & 16.860 & 17.230 & 16.800 & 18.550 \\
\hline $\mathrm{CaO}$ & 24.240 & 23.630 & 23.790 & 21.310 & 23.450 & 23.260 & 22.510 & 23.530 & 21.360 \\
\hline $\mathrm{NiO}$ & 0.038 & 0.060 & 0.042 & 0.078 & 0.057 & 0.048 & 0.060 & 0.064 & 0.065 \\
\hline $\mathrm{Na}_{2} \mathrm{O}$ & 0.054 & 0.085 & 0.067 & 0.083 & 0.081 & 0.053 & 0.060 & 0.082 & 0.044 \\
\hline $\mathrm{K}_{2} \mathrm{O}$ & 0.001 & 0.007 & 0.001 & 0.008 & 0.004 & 0.002 & 0.001 & 0.001 & 0.001 \\
\hline Sum & 100.08 & 99.54 & 99.98 & 100.503 & 100.52 & 98.60 & 99.17 & 99.83 & 99.94 \\
\hline
\end{tabular}

Table 7. Microprobe profiles in two clinopyroxene porphyroclasts from Hole 920D.

\begin{tabular}{|c|c|c|c|c|c|c|c|}
\hline $\begin{array}{l}\text { Core, section, } \\
\text { interval }(\mathrm{cm}) \text { : }\end{array}$ & $15 \mathrm{R}-1,15-20$ & $15 \mathrm{R}-1,15-20$ & $15 \mathrm{R}-1,15-20$ & $15 \mathrm{R}-1,15-20$ & $21 \mathrm{R}-2,74-79$ & $21 \mathrm{R}-2,74-79$ & $21 \mathrm{R}-2,74-79$ \\
\hline $\mathrm{SiO}_{2}$ & 51.41 & 51.71 & 50.17 & 52.39 & 51.01 & 50.51 & 51.13 \\
\hline $\mathrm{TiO}_{2}$ & 0.09 & 0.07 & 0.08 & 0.08 & 0.09 & 0.12 & 0.10 \\
\hline $\mathrm{Al}_{2} \mathrm{O}_{3}$ & 4.96 & 5.47 & 5.48 & 5.15 & 4.83 & 5.68 & 5.20 \\
\hline $\mathrm{Cr}_{2} \mathrm{O}_{3}$ & 1.25 & 1.36 & 1.43 & 1.18 & 1.33 & 1.63 & 1.32 \\
\hline $\mathrm{FeO}$ & 3.14 & 3.27 & 2.97 & 4.27 & 2.96 & 2.58 & 2.57 \\
\hline $\mathrm{MnO}$ & 0.11 & 0.10 & 0.10 & 0.12 & 0.12 & 0.10 & 0.08 \\
\hline $\mathrm{MgO}$ & 17.63 & 17.38 & 17.27 & 22.07 & 17.82 & 16.01 & 16.40 \\
\hline $\mathrm{CaO}$ & 21.92 & 21.19 & 22.85 & 15.48 & 21.71 & 23.83 & 23.91 \\
\hline $\mathrm{NiO}$ & 0.06 & 0.03 & 0.04 & 0.05 & - & - & - \\
\hline $\mathrm{Na}_{2} \mathrm{O}$ & 0.06 & 0.06 & 0.06 & 0.06 & 0.07 & 0.05 & 0.07 \\
\hline $\mathrm{K}_{2} \mathrm{O}$ & 0.00 & 0.00 & 0.00 & 0.01 & 0.00 & 0.00 & 0.00 \\
\hline Sum & 100.63 & 100.64 & 100.45 & 100.86 & 99.94 & 100.51 & 100.77 \\
\hline
\end{tabular}

Note: $-=$ not detected.

thopyroxene grains follows the general foliation in the samples. Kink banding is well expressed. Interstitial olivine is only present in the outermost parts of the mosaics.

Varieties a to c display thin diopside exsolutions, which mostly are parallel to (100) of orthopyroxene, but larger twinned plates also occur with c-axes parallel, but long axes oblique (approximately $30^{\circ}$ to /001/ of orthopyroxene). In orthopyroxene porphyroclasts elongated oblique to $/ 001 /$ the exsolved clinopyroxene strips are not parallel to (100) of orthopyroxene, but follow the elongation of the orthopy- roxene host grain (Pl. 1, Fig. 4). In some instances, columnar plates of exsolved clinopyroxene, up to $0.8 \mathrm{~mm}$ long, obliquely crosscut earlier (100) exsolution lamellae in porphyroclastic orthopyroxenes.

In contrast to the deformed varieties, the polygonal grains along the surface of orthopyroxene porphyroclasts and between clinopyroxene porphyroclasts are not deformed and do not contain exsolution lamellae. A textural gradation between interstitial grains and exsolved enstatite in the adjacent diopside clasts was observed in several thin sections (Pl. 2, Fig. 2). 
Table 8. Microprobe analyses of small deformed clinopyroxene from Hole 920B.

\begin{tabular}{lrrrr}
\hline $\begin{array}{l}\text { Core, section, } \\
\text { interval (cm): }\end{array}$ & 13R-3, 21-26 & 13R-3, 21-26 & 13R-3, 21-26 & 13R-3, 21-26 \\
\hline $\mathrm{SiO}_{2}$ & 52.66 & 51.84 & 50.56 & 51.08 \\
$\mathrm{TiO}_{2}$ & 0.08 & 0.07 & 0.09 & 0.09 \\
$\mathrm{Al}_{2} \mathrm{O}_{3}$ & 4.77 & 5.34 & 5.22 & 5.00 \\
$\mathrm{Cr}_{2} \mathrm{O}_{3}$ & 1.21 & 1.25 & 1.39 & 1.30 \\
$\mathrm{FeO}$ & 4.81 & 4.66 & 2.51 & 2.49 \\
$\mathrm{MnO}$ & 0.10 & 0.09 & 0.08 & 0.10 \\
$\mathrm{MgO}$ & 24.27 & 23.73 & 16.24 & 16.98 \\
$\mathrm{CaO}$ & 13.29 & 12.17 & 23.84 & 23.22 \\
$\mathrm{NiO}$ & 0.07 & 0.08 & 0.07 & 0.06 \\
$\mathrm{Na}{ }_{2} \mathrm{O}$ & 0.04 & 0.03 & 0.07 & 0.08 \\
$\mathrm{~K}_{2} \mathrm{O}$ & 0.01 & 0.00 & 0.00 & 0.00 \\
$\mathrm{Sum}$ & 101.31 & 99.26 & 100.07 & 100.41 \\
\hline
\end{tabular}

\section{Chemistry}

Microprobe analyses of different orthopyroxene varieties are listed in Tables $2-5$. The analyses refer to porphyroclasts (29, including profiles in two grains), small undulatory grains (3), anhedral grains in mosaic aggregates (6), undeformed polygonal grains (4), and exsolution lamellae (1). Most analyses (41) are of samples from Hole 920D. Two analyses from a matrix grain and a polygonal grain in Sample 920B-13R-3, 21-26 cm, are included.

The orthopyroxenes from the first three varieties display variations of enstatite (En) from 84.3 to 88.7 , ferrosilite (Fs) from 8.9 to 10.1 , and wollastonite (Wo) from 1.8 to 6.6. Figure 3 does not show systematic downhole variations of the En content, and the variation in large porphyroclasts nearly exceeds the span of all En values (Fig. 4). Some porphyroclasts have high $\mathrm{CaO}$ contents $(>2.7 \mathrm{wt} \%)$, and are

Table 9. Microprobe analyses of clinopyroxene exsolutions from orthopyroxene, Hole 920D.

\begin{tabular}{|c|c|c|c|c|c|c|c|}
\hline $\begin{array}{l}\text { Core, section, } \\
\text { interval }(\mathrm{cm}) \text { : }\end{array}$ & $18 \mathrm{R}-4,15-20$ & $18 \mathrm{R}-4,15-20$ & $18 \mathrm{R}-4,15-20$ & $22 \mathrm{R}-5,37-43$ & $22 \mathrm{R}-5,37-43$ & $22 R-5,37-43$ & $22 \mathrm{R}-5,37-43$ \\
\hline $\mathrm{SiO}_{2}$ & 51.37 & 51.13 & 50.73 & 51.35 & 51.00 & 51.34 & 50.75 \\
\hline $\mathrm{TiO}_{2}$ & 0.12 & 0.10 & 0.15 & 0.12 & 0.13 & 0.13 & 0.11 \\
\hline $\mathrm{Al}_{2} \mathrm{O}_{3}$ & 4.86 & 5.08 & 4.86 & 4.61 & 4.67 & 4.00 & 5.08 \\
\hline $\mathrm{Cr}_{2} \mathrm{O}_{3}$ & 1.43 & 1.34 & 1.43 & 1.26 & 1.19 & 1.03 & 1.41 \\
\hline $\mathrm{FeO}$ & 2.40 & 2.36 & 2.26 & 2.35 & 2.39 & 2.12 & 2.52 \\
\hline $\mathrm{MnO}$ & 0.07 & 0.09 & 0.07 & 0.08 & 0.05 & 0.08 & 0.08 \\
\hline $\mathrm{MgO}$ & 16.60 & 16.53 & 15.97 & 16.49 & 16.28 & 16.15 & 16.08 \\
\hline $\mathrm{CaO}$ & 23.77 & 23.74 & 24.13 & 20.40 & 23.35 & 24.00 & 23.15 \\
\hline $\mathrm{NiO}$ & - & - & - & - & - & - & - \\
\hline $\mathrm{Na}_{2} \mathrm{O}$ & 0.09 & 0.08 & 0.09 & 0.08 & 0.06 & 0.07 & 0.08 \\
\hline $\mathrm{K}_{2} \mathrm{O}$ & 0.00 & 0.01 & 0.00 & 0.00 & 0.00 & 0.00 & 0.00 \\
\hline Sum & 100.71 & 100.46 & 99.69 & 99.74 & 99.12 & 98.93 & 99.26 \\
\hline $\begin{array}{l}\text { Core, section, } \\
\text { interval }(\mathrm{cm}) \text { : }\end{array}$ & $22 \mathrm{R}-5,37-43$ & $18 \mathrm{R}-4,15-20$ & $18 \mathrm{R}-4,15-20$ & $18 \mathrm{R}-4,15-20$ & $18 \mathrm{R}-4,15-20$ & $18 \mathrm{R}-4,15-20$ & $18 R-4,15-20$ \\
\hline $\mathrm{SiO}_{2}$ & 50.38 & 51.09 & 50.66 & 50.68 & 50.01 & 50.69 & 50.72 \\
\hline $\mathrm{TiO}_{2}^{2}$ & 0.09 & 0.13 & 0.15 & 0.15 & 0.13 & 0.12 & 0.15 \\
\hline $\mathrm{Al}_{2} \mathrm{O}_{3}$ & 4.90 & 5.15 & 5.31 & 5.18 & 5.24 & 5.35 & 5.59 \\
\hline $\mathrm{Cr}_{2} \mathrm{O}_{3}$ & 1.31 & 1.44 & 1.41 & 1.41 & 1.51 & 1.45 & 1.51 \\
\hline $\mathrm{FeO}$ & 2.54 & 2.44 & 2.34 & 2.28 & 2.41 & 2.41 & 2.88 \\
\hline $\mathrm{MnO}$ & 0.10 & 0.05 & 0.06 & 0.05 & 0.09 & 0.08 & 0.09 \\
\hline $\mathrm{MgO}$ & 16.28 & 16.63 & 16.15 & 16.12 & 16.29 & 16.18 & 17.37 \\
\hline $\mathrm{CaO}$ & 22.87 & 23.45 & 23.85 & 23.77 & 23.31 & 23.48 & 21.61 \\
\hline $\mathrm{NiO}$ & - & 0.06 & - & - & 0.08 & - & - \\
\hline $\mathrm{Na}_{2} \mathrm{O}$ & 0.09 & 0.08 & 0.11 & 0.10 & 0.06 & 0.12 & 0.08 \\
\hline $\mathrm{K}_{2} \mathrm{O}$ & 0.01 & 0.00 & 0.01 & 0.00 & 0.00 & 0.00 & 0.00 \\
\hline Sum & 98.57 & 100.52 & 100.05 & 99.74 & 99.13 & 99.88 & 100.00 \\
\hline
\end{tabular}

Notes: Analyses 9-14 are from close to a kink band in orthopyroxene; $-=$ not detected.

Table 10. Microprobe analyses of interstitial clinopyroxene, Hole 920D.

\begin{tabular}{|c|c|c|c|c|c|c|c|c|}
\hline $\begin{array}{l}\text { Core, section, } \\
\text { interval }(\mathrm{cm}) \text { : }\end{array}$ & $18 \mathrm{R}-4,15-20$ & $18 \mathrm{R}-4,15-20$ & $18 \mathrm{R}-4,15-20$ & $18 \mathrm{R}-4,15-20$ & $18 \mathrm{R}-4,15-20$ & $22 \mathrm{R}-5,15-20$ & $22 \mathrm{R}-5,37-43$ & $22 R-5,37-43$ \\
\hline $\mathrm{SiO}_{2}$ & 51.33 & 51.40 & 50.76 & 50.61 & 50.95 & 50.720 & 51.440 & 51.35 \\
\hline $\mathrm{TiO}_{2}^{2}$ & 0.10 & 0.12 & 0.14 & 0.13 & 0.12 & 0.112 & 0.090 & 0.11 \\
\hline $\mathrm{Al}_{2} \mathrm{O}_{3}$ & 4.95 & 4.76 & 5.28 & 5.28 & 5.08 & 4.608 & 4.469 & 4.69 \\
\hline $\mathrm{Cr}_{2} \mathrm{O}_{3}$ & 1.35 & 1.34 & 1.36 & 1.45 & 1.34 & 1.176 & 1.073 & 1.16 \\
\hline $\mathrm{FeO}$ & 2.77 & 2.37 & 2.40 & 2.49 & 2.51 & 2.630 & 2.770 & 2.81 \\
\hline $\mathrm{MnO}$ & 0.09 & 0.06 & 0.08 & 0.08 & 0.09 & 0.100 & 0.090 & 0.09 \\
\hline $\mathrm{MgO}$ & 17.00 & 16.59 & 16.36 & 16.35 & 16.26 & 16.800 & 18.550 & 17.27 \\
\hline $\mathrm{CaO}$ & 22.63 & 23.22 & 24.01 & 23.20 & 23.63 & 23.530 & 21.360 & 21.93 \\
\hline $\mathrm{NiO}$ & - & - & - & - & 0.064 & 0.065 & - & - \\
\hline $\mathrm{Na}_{2} \mathrm{O}$ & 0.09 & 0.08 & 0.10 & 0.10 & 0.09 & 0.082 & 0.044 & 0.09 \\
\hline $\mathrm{K}_{2} \mathrm{O}$ & 0.00 & 0.00 & 0.00 & 0.00 & 0.00 & 0.001 & 0.001 & 0.01 \\
\hline Sum & 100.31 & 99.94 & 100.49 & 99.69 & 100.07 & 99.83 & 99.94 & 99.51 \\
\hline $\begin{array}{l}\text { Core, section, } \\
\text { interval }(\mathrm{cm}) \text { : }\end{array}$ & $22 \mathrm{R}-5,37-43$ & $22 \mathrm{R}-5,37-43$ & $22 \mathrm{R}-5,37-43$ & $22 \mathrm{R}-5,37-43$ & $22 \mathrm{R}-5,37-43$ & $22 \mathrm{R}-5,37-43$ & $22 \mathrm{R}-5,37-43$ & $22 \mathrm{R}-5,37-43$ \\
\hline $\mathrm{SiO}_{2}$ & 51.31 & 51.14 & 50.90 & 51.60 & 50.90 & 50.75 & 52.07 & 50.91 \\
\hline $\mathrm{TiO}_{2}^{2}$ & 0.10 & 0.10 & 0.11 & 0.08 & 0.11 & 0.11 & 0.10 & 0.08 \\
\hline $\mathrm{Al}_{2} \mathrm{O}_{3}$ & 5.12 & 4.85 & 4.82 & 4.53 & 4.26 & 4.66 & 4.63 & 4.99 \\
\hline $\mathrm{Cr}_{2} \mathrm{O}_{3}$ & 1.32 & 1.15 & 1.19 & 1.11 & 1.08 & 1.17 & 1.11 & 1.23 \\
\hline $\mathrm{FeO}$ & 2.61 & 3.02 & 2.71 & 3.18 & 2.37 & 2.69 & 3.35 & 3.16 \\
\hline $\mathrm{MnO}$ & 0.10 & 0.10 & 0.08 & 0.10 & 0.08 & 0.07 & 0.10 & 0.07 \\
\hline $\mathrm{MgO}$ & 16.33 & 17.63 & 16.60 & 18.48 & 16.51 & 16.97 & 18.89 & 18.13 \\
\hline $\mathrm{CaO}$ & 23.59 & 21.22 & 22.63 & 20.73 & 23.13 & 22.36 & 19.89 & 20.22 \\
\hline $\mathrm{NiO}$ & - & - & - & - & - & - & - & - \\
\hline $\mathrm{Na}_{2} \mathrm{O}$ & 0.09 & 0.08 & 0.08 & 0.07 & 0.09 & 0.08 & 0.07 & 0.09 \\
\hline $\mathrm{K}_{2} \mathrm{O}$ & 0.00 & 0.00 & 0.00 & 0.01 & 0.00 & 0.00 & 0.00 & 0.00 \\
\hline Sum & 100.57 & 99.29 & 99.12 & 99.89 & 98.53 & 98.86 & 100.21 & 98.88 \\
\hline
\end{tabular}


Table 11. Microprobe analyses of chromium-bearing spinel.

\begin{tabular}{|c|c|c|c|c|c|c|c|c|}
\hline \multirow{2}{*}{$\begin{array}{l}\text { Hole: } \\
\text { Core, section, } \\
\text { interval }(\mathrm{cm}) \text { : }\end{array}$} & \multicolumn{8}{|c|}{$920 \mathrm{D}$} \\
\hline & $14 \mathrm{R}-3,68-74$ & $15 \mathrm{R}-1,15-20$ & $17 R-4,75-81$ & $21 \mathrm{R}-2,74-79$ & $6 \mathrm{R}-2,100-104$ & $8 R-2,62-69$ & IOR-3, 8-12 & $22 \mathrm{R}-5,37-43$ \\
\hline $\mathrm{Cr}_{2} \mathrm{O}_{3}$ & 26.10 & 27.02 & 26.53 & 27.19 & 27.00 & 25.66 & 30.55 & 25.93 \\
\hline $\mathrm{Al}_{2} \mathrm{O}_{3}$ & 42.20 & 41.81 & 41.85 & 42.00 & 42.58 & 42.20 & 38.94 & 41.97 \\
\hline $\mathrm{Fe}_{2} \mathrm{O}_{3}$ & 2.60 & 1.64 & 2.90 & 1.73 & 1.35 & 2.51 & 1.55 & 2.30 \\
\hline $\mathrm{TiO}_{2}$ & 0.06 & 0.04 & 0.08 & 0.06 & 0.07 & 0.04 & 0.09 & 0.07 \\
\hline $\mathrm{FeO}^{-}$ & 11.52 & 13.08 & 11.32 & 11.78 & 12.09 & 11.90 & 13.02 & 11.34 \\
\hline $\mathrm{MnO}$ & 0.13 & 0.10 & 0.09 & 0.15 & 0.11 & 0.12 & 0.14 & 0.13 \\
\hline $\mathrm{MgO}$ & 17.69 & 16.66 & 17.83 & 17.51 & 17.43 & 17.31 & 16.50 & 17.56 \\
\hline $\mathrm{NiO}$ & 0.20 & 0.21 & 0.22 & 0.23 & 0.26 & 0.21 & 0.19 & 0.24 \\
\hline Sum & 100.50 & 100.56 & 100.64 & 100.64 & 100.89 & 99.95 & 100.98 & 99.55 \\
\hline
\end{tabular}

\begin{tabular}{|c|c|c|c|c|c|c|c|}
\hline \multirow{2}{*}{$\begin{array}{l}\text { Hole: } \\
\text { Core, section, } \\
\text { interval }(\mathrm{cm}) \text { : }\end{array}$} & \multicolumn{4}{|c|}{ 920D } & \multicolumn{3}{|c|}{$920 \mathrm{~B}$} \\
\hline & $18 \mathrm{R}-4,15-20$ & $18 R-4,15-20$ & $21 \mathrm{R}-2,74-79$ & $21 \mathrm{R}-2,74-79$ & $13 R-3,21-26$ & $13 \mathrm{R}-3,21-26$ & $13 R-3,21-26$ \\
\hline $\mathrm{Cr}_{2} \mathrm{O}_{3}$ & 25.25 & 25.51 & 23.50 & 22.10 & 25.65 & 25.82 & 23.12 \\
\hline $\mathrm{Al}_{2} \mathrm{O}_{3}$ & 42.55 & 42.53 & 45.17 & 46.52 & 42.82 & 42.12 & 44.38 \\
\hline $\mathrm{Fe}_{2} \mathrm{O}_{3}$ & 2.24 & 2.07 & 1.45 & 1.14 & 1.44 & 1.84 & 1.52 \\
\hline $\mathrm{TiO}_{2}$ & 0.04 & 0.03 & 0.02 & 0.02 & 0.04 & 0.04 & 0.04 \\
\hline $\mathrm{FeO}^{2}$ & 13.23 & 13.81 & 12.05 & 12.04 & 12.27 & 11.99 & 12.22 \\
\hline $\mathrm{MnO}$ & 0.13 & 0.13 & 0.08 & 0.11 & 0.12 & 0.09 & 0.10 \\
\hline $\mathrm{MgO}$ & 16.69 & 16.38 & 17.11 & 7.75 & 17.13 & 17.19 & 17.52 \\
\hline $\mathrm{NiO}$ & - & - & - & - & 0.22 & 0.21 & 0.18 \\
\hline Sum & 100.13 & 100.47 & 99.96 & 99.68 & 99.73 & 99.36 & 99.39 \\
\hline
\end{tabular}

Note: $-=$ not detected.

therefore classified as pigeonite (numbers 3, 4, 14, 20, 22, 23; Fig. 4). The $\mathrm{CaO}$ content in these porphyroclasts must have been higher originally, because they contain abundant exsolved clinopyroxene.

The orthopyroxene porphyroclasts have generally higher Wo and $\mathrm{Mg} \#\left(\mathrm{Mg} /\left[\mathrm{Mg}+\mathrm{Fe}^{2+}\right]\right)$ than the small individual grains and the angular grains in mosaic aggregates. These compositional differences could reveal that either orthopyroxene porphyroclasts have exsolved less clinopyroxene than smaller orthopyroxene grains or that the smaller grains originated from dismembering of porphyroclasts with extensive clinopyroxene exsolution.

$\mathrm{Al}_{2} \mathrm{O}_{3}$ and $\mathrm{Cr}_{2} \mathrm{O}_{3}$ contents are positively correlated in the investigated orthopyroxene varieties (Fig. 4). Both oxides vary irregularly, independent of the $\mathrm{Mg} \#$ or the En proportions. The highest contents of both oxides were obtained from an exsolved orthopyroxene in clinopyroxene and from a porphyroclast rather devoid of clinopyroxene exsolution lamellae. The lowest contents were found in small individual orthopyroxenes with spinel exsolutions.

$\mathrm{Al}_{2} \mathrm{O}_{3}, \mathrm{Cr}_{2} \mathrm{O}_{3}, \mathrm{TiO}_{2}$, and $\mathrm{NiO}$ contents in the orthopyroxene porphyroclasts markedly decrease with increasing exsolution of clinopyroxene. Their cores consistently contain more $\mathrm{Al}_{2} \mathrm{O}_{3}, \mathrm{Cr}_{2} \mathrm{O}_{3}$, and $\mathrm{NiO}$ than their rims. This feature was already observed in porphyroclasts from ophiolitic and abyssal peridotite tectonites by previous authors (Sinton, 1977; Hamlyn and Bonatti, 1981; Quick, 1981; Cannat et al., 1990) and was attributed by these authors to reequilibration. The impoverishment of both oxides in the rims of our investigated porphyroclasts cannot be related to the exsolution of spinel, which is absent in or near the analyzed grains.

\section{Clinopyroxene}

Clinopyroxene is present in the investigated peridotites as the following morphological varieties:

a. Anhedral, weakly elongated, large (up to $4 \mathrm{~mm}$ ) grains (including porphyroclasts). These grains contain strain lamellae and less common enstatite exsolutions parallel to (100) (Pl. 2, Fig. 2).

b. Polycrystalline aggregates of interlocked, large ( 0.6 to 2 $\mathrm{mm}$ ) grains.

c. Aggregates of undeformed grains $(0.4$ to $0.6 \mathrm{~mm})$ between deformed clinopyroxene porphyroclasts. In these aggre- gates polygonal olivine, enstatite, and occasionally spinel can be associated.

d. Exsolution lamellae parallel to (100) of orthopyroxene and exsolution plates with long axes oblique to $/ 001 /$ of orthopyroxene (Pl. 1, Figs. 1, 3; Pl. 2, Fig. 3).

e. Interstitial undeformed crystals $(0.1-0.5 \mathrm{~mm})$ that are devoid of exsolutions and often twinned (Pl. 1, Fig. 3).

The first two varieties are comparable to the enstatite porphyroclasts and the aggregates of undeformed polygonal orthopyroxene. The interstitial clinopyroxene is closely associated with deformed enstatite. It occurs (1) in the interstices of mosaic aggregates of anhedral enstatite (PI. 1, Fig. 3), (2) along the lobate outlines of enstatite porphyroclasts (Pl. 1, Fig. 1), (3) between deformed enstatite and olivine (Pl. 2, Fig. 3), and (4) as overgrowths on olivine inclusions in porphyroclastic enstatite. The rarely encountered clinopyroxene outlining embayments of spinel probably belongs to this variety.

The (100) exsolution lamellae and platy exsolutions of clinopyroxene in porphyroclastic orthopyroxene often proceed with optical continuity into 0.15 -mm-thick plates along opening kink bands of orthopyroxene (Pl. 2, Figs. 1, 3), which apparently present a favorable site for the migration of exsolved clinopyroxene. In an advanced stage of disintegration, the space between the orthopyroxene fragments was occupied by neoblasts of (originally exsolved) clinopyroxene. They can be accompanied by olivine and orthopyroxene neoblasts.

\section{Chemistry}

Fifty-nine microprobe analyses from 54 grains in 10 sections are listed in Tables 6-10.29 analyses refer to the morphological variety a (porphyroclasts: 25; small deformed grains: 4 ), 14 analyses are from exsolutions in deformed orthopyroxene and 16 analyses refer to undeformed interstitial clinopyroxene. All analyses with the exception of the small deformed grains are from samples of Hole 920D.

The clinopyroxene porphyroclasts and the small deformed grains have molar proportions of Wo between 41.8 and 49.5, En between 46.2 and 50.4 , and Fs between 3.4 and 5.4 (diopside to augite). They display a markedly wider compositional range than deformed orthopyroxenes. This feature is reflected by the large within-grain and grain-to-grain chemical variations, which are in part responsible for the irregularity of the vertical variation of the En content in Figure 3. As with orthopyroxenes, the main variations in these proportions take 
Table 12. Chemical composition of serpentinized peridotites.

\begin{tabular}{|c|c|c|c|c|c|c|c|c|c|c|c|c|c|c|c|c|}
\hline $\begin{array}{l}\text { Core, section, } \\
\text { interval }(\mathrm{cm})\end{array}$ & $\begin{array}{l}\mathrm{SiO}_{2}^{*} \\
(\%)\end{array}$ & $\begin{array}{l}\mathrm{TiO}_{2} * \\
(\%)\end{array}$ & $\begin{array}{c}\mathrm{Al}_{2} \mathrm{O}_{3}{ }^{*} \\
(\%)\end{array}$ & $\begin{array}{c}\mathrm{Fe}_{2} \mathrm{O}_{3}{ }^{*} \\
(\%)\end{array}$ & $\begin{array}{c}\mathrm{MnO}^{*} \\
(\%)\end{array}$ & $\begin{array}{l}\mathrm{MgO}^{*} \\
(\%)\end{array}$ & $\begin{array}{l}\mathrm{CaO}^{*} \\
(\%)\end{array}$ & $\begin{array}{c}\mathrm{Na}_{2} \mathrm{O}^{*} \\
(\%)\end{array}$ & $\begin{array}{c}\left(\mathrm{SO}_{3}\right)^{*} \\
(\%)\end{array}$ & $\begin{array}{l}\text { LOI* } \\
(\%)\end{array}$ & As** & $\mathrm{Ba}^{* *}$ & $\mathrm{Ce}^{* *}$ & $\mathrm{Co}^{*}$ & & \\
\hline \multicolumn{17}{|l|}{ Hole 920B } \\
\hline IW-3, 55-61 & 38.68 & 0.018 & 1.28 & 8.04 & 0.12 & 37.72 & 0.10 & 0.02 & 0.29 & 13.08 & 0.50 & 1.41 & 0.022 & 100 & & \\
\hline $2 \mathrm{R}-1,13-17$ & 38.45 & 0.015 & 1.08 & 8.17 & 0.10 & 37.48 & 0.19 & 0.05 & 0.44 & 13.34 & 0.31 & 0.67 & 0.059 & 102 & & \\
\hline $3 \mathrm{R}-1,21-26$ & 38.68 & 0.017 & 1.04 & 8.11 & 0.10 & 37.51 & 0.11 & 0.15 & 0.36 & 13.38 & 0.24 & 0.63 & 0.140 & 107 & & \\
\hline $4 \mathrm{R}-1,64-68$ & 39.92 & 0.023 & 1.42 & 7.86 & 0.11 & 37.37 & 0.31 & 0.06 & 0.29 & 12.11 & 0.24 & 1.12 & 0.039 & 98 & & \\
\hline 5R-1, 106-111 & 38.92 & 0.016 & 1.02 & 7.88 & 0.10 & 37.51 & 0.29 & 0.02 & 0.31 & 13.27 & 0.42 & 0.28 & 0.018 & 95 & & \\
\hline $6 \mathrm{R}-3,13-18$ & 40.14 & 0.015 & 0.97 & 8.07 & 0.09 & 37.37 & 0.15 & 0.06 & 0.25 & 12.20 & 0.00 & 0.42 & 0.017 & 92 & & \\
\hline $7 \mathrm{R}-2,50-55$ & 39.08 & 0.020 & 1.20 & 7.72 & 0.10 & 37.50 & 0.12 & 0.02 & 0.24 & 13.26 & 0.00 & 0.71 & 0.022 & 104 & & \\
\hline $8 \mathrm{R}-2,78-82$ & 39.82 & 0.020 & 1.17 & 7.73 & 0.11 & 37.63 & 0.28 & 0.02 & 0.18 & 12.44 & 0.43 & 0.25 & 0.160 & 101 & & \\
\hline $9 \mathrm{R}-2,92-97$ & 40.65 & 0.027 & 1.61 & 7.55 & 0.11 & 37.12 & 0.47 & 0.02 & 0.20 & 11.45 & 0.33 & 0.65 & 0.053 & 95 & & \\
\hline $10 \mathrm{R}-1,40-45$ & 39.57 & 0.019 & 1.24 & 8.16 & 0.11 & 37.32 & 0.33 & 0.05 & 0.20 & 12.56 & 0.00 & 0.48 & 0.160 & 97 & & \\
\hline IIR-1, 50-53 & 38.85 & 0.017 & 1.33 & 7.91 & 0.11 & 37.69 & 0.11 & 0.02 & 0.36 & 12.97 & 0.36 & 0.21 & 0.036 & 96 & & \\
\hline $12 \mathrm{R}-1,96-100$ & 38.64 & 0.016 & 1.17 & 8.01 & 0.11 & 37.84 & 0.66 & 0.02 & 0.18 & 12.87 & 0.27 & 0.25 & 0.022 & 104 & & \\
\hline $13 \mathrm{R}-3,21-26$ & 39.87 & 0.019 & 1.36 & 7.80 & 0.13 & 37.63 & 0.73 & 0.02 & 0.23 & 11.72 & 0.90 & 1.78 & 0.016 & 98 & & \\
\hline \multicolumn{17}{|l|}{ Hole 920D } \\
\hline $2 \mathrm{R}-1,29-36$ & 39.44 & 0.016 & 0.82 & 8.23 & 0.11 & 37.16 & 0.37 & 0.02 & 0.37 & 13.05 & 0.23 & 0.51 & 0.630 & 103 & & \\
\hline $3 \mathrm{R}-1,110-115$ & 39.90 & 0.021 & 1.55 & 7.40 & 0.12 & 37.10 & 0.34 & 0.06 & 0.32 & 12.52 & 0.79 & 0.22 & 0.011 & 98 & & \\
\hline $4 \mathrm{R}-2,100-104$ & 40.20 & 0.021 & 1.46 & 7.51 & 0.11 & 37.15 & 0.27 & 0.02 & 0.22 & 12.27 & 0.59 & 0.41 & 0.012 & 97 & & \\
\hline SR-1,49-55 & 38.91 & 0.017 & 1.25 & 7.91 & 0.12 & 37.70 & 0.18 & 0.02 & 0.31 & 12.99 & 0.35 & $\begin{array}{l}0.41 \\
0.19\end{array}$ & 0.034 & 101 & & \\
\hline $6 \mathrm{R}-2,100-104$ & 39.33 & 0.019 & 1.34 & 8.08 & 0.10 & 37.56 & 0.16 & 0.02 & 0.29 & 12.50 & 0.76 & 0.26 & 0.120 & 98 & & \\
\hline $7 \mathrm{R}-2,67-71$ & 40.08 & 0.022 & 1.42 & 7.91 & 0.11 & 37.24 & 0.32 & 0.02 & 0.27 & 12.03 & 0.87 & 0.25 & 0.100 & 102 & & \\
\hline $8 \mathrm{R}-2,62-69$ & 39.67 & 0.021 & 1.33 & 7.97 & 0.11 & 37.47 & 0.28 & 0.02 & 0.30 & 12.30 & 0.32 & 0.23 & 0.080 & 101 & & \\
\hline 10R-3, 8-12 & 39.17 & 0.022 & 1.44 & 8.18 & 0.10 & 37.46 & 0.23 & 0.02 & 0.26 & 12.23 & 0.75 & 0.42 & 0.040 & 96 & & \\
\hline $11 \mathrm{R}-1,102-107$ & 38.72 & 0.029 & 0.96 & 8.11 & 0.13 & 37.81 & 0.06 & 0.06 & 0.28 & 13.25 & 1.54 & 1.13 & 0.048 & 101 & & \\
\hline $12 \mathrm{R}-1,60-66$ & 39.78 & 0.015 & 0.87 & 8.25 & 0.12 & 37.20 & 0.05 & 0.02 & 0.23 & 12.94 & 0.94 & 0.62 & 0.089 & 93 & & \\
\hline $13 R-2,20-25$ & 37.84 & 0.016 & 1.20 & 8.02 & 0.11 & 37.38 & 0.71 & 0.02 & 0.29 & 13.75 & 0.74 & 0.38 & 0.078 & 101 & & \\
\hline $14 \mathrm{R}-3,68-74$ & 39.17 & 0.016 & 1.20 & 8.24 & 0.12 & 38.84 & 0.98 & 0.02 & 0.10 & 10.73 & 0.30 & 0.19 & 0.010 & 110 & & \\
\hline $15 \mathrm{R}-1,15-20$ & 37.99 & 0.015 & 1.15 & 8.02 & 0.12 & 37.96 & $\begin{array}{l}1.09 \\
\end{array}$ & 0.02 & 0.13 & 12.76 & 0.74 & 0.47 & 0.029 & 104 & & \\
\hline $16 \mathrm{R}-5,27-32$ & 39.05 & 0.018 & 1.20 & 7.77 & 0.12 & 38.05 & 0.56 & 0.08 & 0.20 & 12,34 & 0.57 & 1.01 & 0.017 & 103 & & \\
\hline $17 \mathrm{R}-4,75-81$ & 38.98 & 0.018 & 1.21 & 7.83 & 0.12 & 37.96 & 0.48 & 0.06 & 0.19 & 12.55 & 0.45 & 0.64 & 0.052 & 100 & & \\
\hline $18 R-4,15-20$ & 39.21 & 0.020 & 1.18 & 8.17 & 0.10 & 37.57 & 0.18 & 0.02 & 0.28 & 12.64 & 0.83 & 0.59 & 0.160 & 104 & & \\
\hline $19 \mathrm{R}-2,46-51$ & 39.14 & 0.018 & 1.28 & 8.19 & 0.10 & 37.33 & 0.31 & 0.02 & 0.45 & 12.58 & 0.19 & 0.54 & 0.160 & 110 & & \\
\hline $20 \mathrm{R}-2,33-48$ & 38.74 & 0.020 & 1.24 & 7.99 & 0.12 & 37.52 & 0.19 & 0.02 & 0.33 & 13.18 & 0.44 & $\begin{array}{l}1.09 \\
\end{array}$ & $\begin{array}{l}0.100 \\
0.210\end{array}$ & 106 & & \\
\hline $21 \mathrm{R}-2,74-79$ & 37.73 & 0.014 & 1.05 & 7.95 & 0.12 & 38.26 & 0.32 & 0.02 & 0.22 & 13.63 & 0.38 & 0.46 & 0.032 & 98 & & \\
\hline $22 \mathrm{R}-5,37-43$ & 39.20 & 0.020 & 1.29 & 7.69 & 0.12 & 37.47 & 0.47 & 0.02 & 0.26 & 12.78 & 0.50 & 0.71 & 0.033 & 98 & & \\
\hline $22 \mathrm{R}-6,18-24$ & 38.04 & 0.016 & 1.24 & 8.04 & 0.10 & 37.56 & 0.18 & 0.02 & 0.66 & 13.49 & 0.51 & 0.68 & 0.047 & 104 & & \\
\hline $\begin{array}{l}\text { Core, section, } \\
\text { interval }(\mathrm{cm})\end{array}$ & $\underset{(\mathrm{ppm})}{\mathrm{Cr}^{*}}$ & $\begin{array}{l}\text { Cs** } \\
\text { (ppm) }\end{array}$ & $\begin{array}{l}\mathrm{Cu}^{*} \\
(\mathrm{ppm})\end{array}$ & $\begin{array}{l}\text { Dy** } \\
(\mathrm{ppm})\end{array}$ & $\begin{array}{l}\mathrm{Er}^{* *} \\
(\mathrm{ppm})\end{array}$ & $\begin{array}{l}\mathrm{Eu}^{* *} \\
(\mathrm{ppm})\end{array}$ & $\begin{array}{l}\mathrm{Gd}^{* *} \\
(\mathrm{ppm})\end{array}$ & $\begin{array}{l}\mathrm{Hf} * * \\
(\mathrm{ppm})\end{array}$ & $\begin{array}{l}\mathrm{Ho} * * \\
(\mathrm{ppm})\end{array}$ & $\begin{array}{l}\mathrm{La}^{* *} \\
(\mathrm{ppm})\end{array}$ & $\begin{array}{l}\mathrm{Lu}^{*} \\
(\mathrm{ppm})\end{array}$ & $\begin{array}{l}\text { *Mo** } \\
(\mathrm{ppm})\end{array}$ & $\begin{array}{l}\mathrm{Nb}^{* * *} \\
(\mathrm{ppm})\end{array}$ & $\begin{array}{l}\mathrm{Nd}^{* * *} \\
(\mathrm{ppm})\end{array}$ & $\begin{array}{l}\mathrm{Ni}^{\mathrm{N} * *} \\
\text { (ppm) }\end{array}$ & $\begin{array}{l}\mathrm{Pr}^{* *} \\
(\mathrm{ppm})\end{array}$ \\
\hline IW-3, 55-61 & 2636 & 0.001 & $<10$ & 0.039 & 0.039 & 0.002 & 0.016 & 0.260 & 0.011 & 0.011 & 0.011 & 0.096 & 7.25 & 0.001 & 2224 & 0.002 \\
\hline $2 \mathrm{R}-1,13-17$ & 2304 & 0.004 & $<10$ & 0.042 & 0.041 & 0.006 & 0.019 & 0.099 & 0.012 & 0.022 & 0.012 & 0.120 & 4.87 & 0.013 & 2226 & 0.005 \\
\hline $3 \mathrm{R}-1,21-26$ & 1753 & 0.005 & 62 & 0.051 & 0.047 & 0.006 & 0.032 & 0.077 & 0.013 & 0.054 & 0.011 & 0.240 & $\begin{array}{l}4.07 \\
4.08\end{array}$ & 0.066 & 2309 & 0.017 \\
\hline $4 \mathrm{R}-1,64-68$ & 2779 & 0.001 & 47 & 0.074 & 0.072 & 0.009 & 0.039 & 0.054 & 0.020 & 0.013 & 0.017 & 0.130 & 3.27 & 0.016 & 2208 & 0.005 \\
\hline $5 \mathrm{R}-1,106-111$ & 1641 & 0.001 & $<10$ & 0.034 & 0.039 & 0.025 & 0.016 & 0.044 & 0.011 & 0.005 & 0.011 & 0.210 & 2.88 & 0.003 & 2203 & 0.001 \\
\hline $6 \mathrm{R}-3,13-18$ & $\begin{array}{l}1071 \\
1770\end{array}$ & 0.003 & 19 & $\begin{array}{l}0.043 \\
0.043\end{array}$ & 0.045 & 0.017 & 0.019 & 0.040 & 0.012 & 0.009 & 0.011 & 0.120 & 3.01 & 0.000 & 2265 & 0.001 \\
\hline $7 \mathrm{R}-2,50-55$ & 2465 & 0.000 & $<10$ & 0.055 & 0.054 & 0.002 & 0.023 & 0.024 & 0.014 & 0.006 & 0.015 & 0.060 & 2.06 & 0.000 & 2235 & 0.001 \\
\hline $8 \mathrm{R}-2,78-82$ & 2256 & 0.000 & $<10$ & 0.046 & 0.048 & 0.020 & 0.024 & 0.020 & 0.012 & 0.053 & 0.014 & 0.070 & 1.64 & 0.060 & 2126 & 0.020 \\
\hline $9 \mathrm{R}-2,92-97$ & 3258 & 0.002 & $<10$ & 0.072 & 0.067 & 0.009 & 0.035 & 0.034 & 0.020 & 0.016 & 0.017 & 0.180 & 1.79 & 0.033 & 2088 & 0.008 \\
\hline $10 \mathrm{R}-1,40-45$ & 2306 & 0.000 & $<10$ & 0.082 & 0.074 & 0.032 & 0.056 & 0.022 & 0.020 & 0.044 & 0.017 & 0.080 & 2.06 & 0.120 & 2102 & 0.026 \\
\hline $11 R-1,50-53$ & 2865 & 0.000 & 16 & 0.046 & 0.049 & 0.006 & 0.019 & 0.017 & 0.012 & 0.016 & 0.014 & 0.080 & 2.02 & 0.005 & 2174 & 0.003 \\
\hline $12 \mathrm{R}-1,96-100$ & 2167 & 0.001 & $<10$ & 0.048 & 0.047 & 0.005 & 0.017 & 0.083 & 0.013 & 0.007 & 0.013 & 0.120 & 2.68 & 0.007 & 2185 & 0.001 \\
\hline $13 \mathrm{R}-3,21-26$ & 2565 & 0.000 & $<10$ & 0.053 & 0.056 & 0.002 & 0.016 & 0.046 & 0.015 & 0.010 & 0.017 & 0.240 & 2.67 & 0.004 & 2118 & 0.001 \\
\hline $2 \mathrm{R}-1,29-36$ & 1712 & 0.004 & $<10$ & 0.130 & 0.094 & 0.250 & 0.120 & 0.058 & 0.029 & 0.260 & 0.019 & 0.180 & 1.89 & 0.380 & 2339 & 0.009 \\
\hline $3 \mathrm{R}-1,110-115$ & 2777 & 0.003 & $<10$ & 0.068 & 0.065 & 0.005 & 0.027 & 0.017 & 0.019 & 0.004 & 0.019 & 0.250 & 1.30 & 0.004 & 2132 & 0.001 \\
\hline $4 \mathrm{R}-2,100-104$ & 2938 & 0.003 & 24 & 0.065 & 0.062 & 0.017 & 0.023 & 0.020 & 0.018 & 0.008 & 0.018 & 0.090 & 1.89 & 0.005 & 2102 & 0.002 \\
\hline $5 \mathrm{R}-1,49-55$ & 2614 & 0.001 & 13 & 0.044 & 0.046 & 0.001 & 0.015 & 0.020 & 0.012 & 0.006 & 0.014 & 0.100 & $\begin{array}{l}1.69 \\
1.96\end{array}$ & 0.005 & 2210 & 0.002 \\
\hline $6 \mathrm{R}-2,100-104$ & 3022 & 0.001 & 17 & 0.059 & 0.052 & 0.017 & 0.028 & 0.033 & 0.014 & 0.043 & 0.015 & 0.170 & 1.72 & 0.046 & 2221 & 0.013 \\
\hline $7 \mathrm{R}-2,67-71$ & 2625 & 0.002 & 15 & 0.073 & 0.064 & 0.003 & 0.028 & 0.020 & 0.018 & 0.008 & 0.018 & 0.090 & 1.67 & 0.007 & 2160 & 0.002 \\
\hline $8 R-2,62-69$ & 2556 & 0.002 & 26 & 0.064 & 0.053 & 0.008 & 0.021 & 0.031 & 0.015 & 0.012 & 0.014 & 0.150 & 1.50 & 0.019 & 2198 & 0.004 \\
\hline $10 \mathrm{R}-3,8-12$ & 4336 & 0.004 & 35 & 0.057 & 0.049 & 0.005 & 0.027 & 0.110 & 0.015 & 0.013 & 0.013 & 0.100 & 3.93 & 0.012 & 2173 & 0.004 \\
\hline $11 R-1,102-107$ & 2370 & 0.002 & 28 & 0.078 & 0.062 & 0.008 & 0.043 & 0.047 & 0.019 & 0.014 & 0.017 & 0.070 & 2.18 & 0.037 & 2072 & 0.006 \\
\hline $12 \mathrm{R}-1,60-66$ & 1631 & 0.006 & $<10$ & 0.059 & 0.052 & 0.110 & 0.034 & 0.021 & 0.015 & 0.030 & 0.016 & 0.060 & 1.65 & 0.067 & 2075 & 0.013 \\
\hline $13 R-2,20-25$ & 2642 & 0.002 & 17 & 0.051 & 0.048 & 0.002 & 0.019 & 0.017 & 0.014 & 0.036 & 0.014 & 0.080 & 1.48 & 0.021 & 2207 & 0.008 \\
\hline $14 \mathrm{R}-3,68-74$ & 2633 & 0.001 & 14 & 0.042 & 0.042 & 0.001 & 0.011 & 0.012 & 0.011 & 0.006 & 0.013 & 0.110 & 1.57 & 0.002 & 2318 & 0.001 \\
\hline
\end{tabular}


Table 12 (continued).

\begin{tabular}{|c|c|c|c|c|c|c|c|c|c|c|c|c|c|c|c|c|}
\hline $\begin{array}{l}\text { Core, section, } \\
\text { interval }(\mathrm{cm})\end{array}$ & $\begin{array}{l}\mathrm{Cr}^{*} \\
(\mathrm{ppm})\end{array}$ & $\begin{array}{l}\mathrm{Cs}^{* *} \\
(\mathrm{ppm})\end{array}$ & $\begin{array}{l}\mathrm{Cu}^{*} \\
(\mathrm{ppm})\end{array}$ & $\begin{array}{l}\text { Dy** } \\
(\mathrm{ppm})\end{array}$ & $\begin{array}{c}\mathrm{Er}^{* *} \\
(\mathrm{ppm})\end{array}$ & $\begin{array}{l}\mathrm{Eu}^{* *} \\
(\mathrm{ppm})\end{array}$ & $\begin{array}{l}\mathrm{Gd}^{* *} \\
(\mathrm{ppm})\end{array}$ & $\begin{array}{l}\mathrm{Hf}^{* * *} \\
(\mathrm{ppm})\end{array}$ & $\begin{array}{l}\mathrm{Ho}^{* *} \\
(\mathrm{ppm})\end{array}$ & $\begin{array}{l}\mathrm{La}^{* *} \\
(\mathrm{ppm})\end{array}$ & $\begin{array}{l}\mathrm{Lu}^{*} \\
(\mathrm{ppm})\end{array}$ & $\begin{array}{l}{ }^{*} \mathrm{Mo}^{* *} \\
(\mathrm{ppm})\end{array}$ & $\begin{array}{l}\mathrm{Nb}^{* *} \\
(\mathrm{ppm})\end{array}$ & $\begin{array}{l}\mathrm{Nd}^{* *} \\
(\mathrm{ppm})\end{array}$ & $\begin{array}{l}\mathrm{Ni}^{* * *} \\
(\mathrm{ppm})\end{array}$ & $\begin{array}{l}\text { Pr** } \\
\text { (ppm) }\end{array}$ \\
\hline $15 \mathrm{R}-1,15-20$ & 2582 & 0.004 & 12 & 0.047 & 0.044 & 0.002 & 0.019 & 0.017 & 0.014 & 0.014 & 0.013 & 0.170 & 1.79 & 0.007 & 2251 & 0.003 \\
\hline $16 \mathrm{R}-5,27-32$ & 2260 & 0.001 & 16 & 0.055 & 0.047 & 0.002 & 0.018 & 0.012 & 0.014 & 0.006 & 0.015 & 0.110 & 1.63 & 0.007 & 2204 & 0.001 \\
\hline $17 \mathrm{R}-4,75-81$ & 2418 & 0.002 & $<10$ & 0.056 & 0.052 & 0.002 & 0.021 & 0.011 & 0.015 & 0.024 & 0.015 & 0.090 & 1.54 & 0.020 & 2147 & 0.005 \\
\hline $18 \mathrm{R}-4,15-20$ & 2366 & 0.001 & 17 & 0.071 & 0.057 & 0.018 & 0.031 & 0.014 & 0.017 & 0.056 & 0.015 & 0.070 & 1.29 & 0.057 & 2280 & 0.015 \\
\hline $19 R-2,46-51$ & 2783 & 0.002 & 15 & 0.0406 & 0.046 & 0.020 & 0.021 & 0.011 & 0.013 & 0.057 & 0.013 & 0.100 & 0.99 & 0.054 & 2214 & 0.018 \\
\hline $20 \mathrm{R}-2,33-48$ & 2421 & 0.009 & $<10$ & 0.068 & 0.057 & 0.015 & 0.038 & 0.028 & 0.017 & 0.081 & 0.017 & 0.160 & 1.28 & 0.089 & 2195 & 0.024 \\
\hline $2 \mathrm{IR}-2,74-79$ & 2182 & 0.004 & 14 & 0.035 & 0.036 & 0.002 & 0.012 & 0.012 & 0.009 & 0.012 & 0.011 & 0.090 & 1.42 & 0.010 & 2265 & 0.003 \\
\hline $22 \mathrm{R}-5,37-43$ & 2265 & 0.002 & 13 & 0.052 & 0.051 & 0.003 & 0.020 & 0.014 & 0.015 & 0.013 & 0.017 & 0.120 & 1.41 & 0.012 & 2139 & 0.003 \\
\hline $22 \mathrm{R}-6,18-24$ & 2449 & 0.004 & 16 & 0.031 & 0.036 & 0.002 & 0.011 & 0.011 & 0.010 & 0.024 & 0.012 & 0.130 & 1.45 & 0.018 & 2183 & 0.005 \\
\hline $\begin{array}{l}\text { Core, section, } \\
\text { interval }(\mathrm{cm})\end{array}$ & $\begin{array}{l}\mathrm{Rb}^{* *} \\
(\mathrm{ppm})\end{array}$ & $\begin{array}{l}\mathrm{Sb}^{* *} \\
(\mathrm{ppm})\end{array}$ & $\begin{array}{l}\mathrm{Sc}^{* * *} \\
(\mathrm{ppm})\end{array}$ & $\begin{array}{l}\mathrm{Sm}^{* *} \\
(\mathrm{ppm})\end{array}$ & $\begin{array}{l}\mathrm{Sr}^{* * *} \\
(\mathrm{ppm})\end{array}$ & $\begin{array}{l}\mathrm{Ta}^{* *} \\
(\mathrm{ppm})\end{array}$ & $\begin{array}{l}\mathrm{Tb}^{* *} \\
(\mathrm{ppm})\end{array}$ & $\begin{array}{l}\mathrm{Th}^{* * *} \\
(\mathrm{ppm})\end{array}$ & $\begin{array}{l}\mathrm{Tm} * * \\
(\mathrm{ppm})\end{array}$ & $\begin{array}{c}\mathrm{U}^{* *} \\
(\mathrm{ppm})\end{array}$ & $\begin{array}{c}\mathrm{V}^{* * *} \\
(\mathrm{ppm})\end{array}$ & $\begin{array}{l}\mathrm{W}^{* *} \\
(\mathrm{ppm})\end{array}$ & $\begin{array}{c}\mathrm{Y}^{* * *} \\
(\mathrm{ppm})\end{array}$ & $\begin{array}{l}\text { Yb** } \\
\text { (ppm) }\end{array}$ & $\begin{array}{c}\mathrm{Zn}^{*} \\
(\mathrm{ppm})\end{array}$ & $\begin{array}{c}\mathrm{Zr}^{*} \\
(\mathrm{ppm})\end{array}$ \\
\hline $1 \mathrm{~W}-3,55-61$ & 0.041 & 0.25 & 10.4 & 0.000 & 0.8 & 9.39 & 0.003 & 0.200 & 0.006 & 0.00 & 35 & 0.320 & 0.35 & 0.063 & 46 & 0.770 \\
\hline $2 \mathrm{R}-1,13-17$ & 0.130 & 0.54 & 9.5 & 0.003 & 30.7 & 6.83 & 0.003 & 0.059 & 0.008 & 0.00 & 38 & 0.870 & 0.40 & 0.068 & 47 & 0.310 \\
\hline $3 \mathrm{R}-1,21-26$ & 0.110 & 0.25 & 9.0 & 0.020 & 2.7 & 5.73 & 0.005 & 0.037 & 0.008 & 0.26 & 41 & 0.200 & 0.41 & 0.069 & 37 & 0.490 \\
\hline $4 \mathrm{R}-1,64-68$ & 0.081 & 0.37 & 11.9 & 0.011 & 11.5 & 4.67 & 0.008 & 0.019 & 0.011 & 0.00 & 55 & 0.088 & 0.63 & 0.098 & 45 & 0.290 \\
\hline $5 \mathrm{R}-1,106-111$ & 0.039 & 0.22 & 8.4 & 0.000 & 46.2 & 4.15 & 0.003 & 0.013 & 0.006 & 0.00 & 30 & 0.520 & 0.35 & 0.064 & 38 & 0.120 \\
\hline $6 \mathrm{R}-3,13-18$ & 0.063 & 0.24 & 9.4 & 0.000 & 0.9 & 4.35 & 0.004 & 0.010 & 0.007 & 0.00 & 36 & 0.140 & 0.39 & 0.067 & 35 & 0.110 \\
\hline $7 \mathrm{R}-2,50-55$ & 0.046 & 0.17 & 10.3 & 0.000 & 1.0 & 2.93 & 0.004 & 0.003 & 0.010 & 0.00 & 40 & 0.470 & 0.48 & 0.079 & 45 & 0.120 \\
\hline $8 R-2,78-82$ & 0.046 & 0.16 & 10.6 & 0.008 & 1.2 & 2.41 & 0.004 & 0.002 & 0.008 & 0.00 & 47 & 0.330 & 0.43 & 0.079 & 40 & 0.130 \\
\hline $9 \mathrm{R}-2,92-97$ & 0.065 & 0.21 & 12.9 & 0.011 & 1.7 & 2.57 & 0.008 & 0.002 & 0.012 & 0.00 & 61 & 0.260 & 0.62 & 0.100 & 46 & 0.430 \\
\hline $10 \mathrm{R}-1,40-45$ & 0.014 & 0.17 & 10.5 & 0.033 & 0.9 & 3.03 & 0.009 & 0.009 & 0.011 & 0.00 & 50 & 0.140 & 0.67 & 0.100 & 41 & 0.220 \\
\hline $11 \mathrm{R}-1,50-53$ & 0.033 & 0.14 & $\begin{array}{l}10.9 \\
10.9\end{array}$ & 0.000 & 1.2 & 3.07 & 0.004 & 0.000 & 0.009 & 0.00 & 53 & 0.320 & 0.43 & 0.076 & $\begin{array}{l}41 \\
46\end{array}$ & 0.110 \\
\hline $12 \mathrm{R}-1,96-100$ & 0.047 & 0.19 & 10.4 & 0.003 & 1.0 & 3.83 & 0.004 & 0.034 & 0.008 & 0.01 & 45 & 1.830 & 0.42 & 0.075 & 40 & 0.200 \\
\hline $13 \mathrm{R}-3,21-26$ & 0.030 & 0.24 & 11.9 & 0.000 & 0.7 & 4.20 & 0.004 & 0.012 & 0.009 & 0.00 & 53 & 3.700 & 0.49 & 0.087 & 45 & 0.230 \\
\hline $2 \mathrm{R}-1,29-36$ & 0.094 & 0.18 & 7.0 & 0.100 & 57.8 & 2.84 & 0.019 & 0.010 & 0.013 & 0.00 & 35 & 0.840 & 1.02 & 0.110 & 43 & 1.290 \\
\hline $3 R-1,110-115$ & 0.071 & 0.18 & 13.7 & 0.000 & 9.9 & 1.92 & 0.006 & 0.002 & 0.011 & 0.00 & 56 & 17.400 & 0.60 & 0.100 & 43 & 0.070 \\
\hline $4 \mathrm{R}-2,100-104$ & 0.091 & 0.17 & 12.9 & 0.004 & 1.8 & 2.98 & 0.006 & 0.004 & 0.011 & 0.00 & 58 & 1.280 & 0.57 & 0.097 & 50 & 0.100 \\
\hline $5 \mathrm{R}-1,49-55$ & 0.042 & 0.18 & 10.5 & 0.000 & 10.7 & 3.15 & 0.004 & 0.004 & 0.008 & 0.01 & 45 & 0.140 & 0.41 & 0.074 & 51 & 0.110 \\
\hline $6 \mathrm{R}-2,100-104$ & 0.070 & 0.33 & 10.2 & 0.012 & 1.0 & 2.78 & 0.005 & 0.005 & 0.010 & 0.03 & 53 & 0.130 & 0.49 & 0.087 & 56 & 0.600 \\
\hline $7 \mathrm{R}-2,67-71$ & 0.068 & 0.22 & 12.5 & 0.002 & 1.2 & 2.81 & 0.007 & 0.003 & 0.011 & 0.00 & 60 & 0.230 & 0.60 & 0.098 & 34 & 0.240 \\
\hline $8 \mathrm{R}-2,62-69$ & 0.059 & 0.24 & 10.3 & 0.005 & 2.1 & 2.51 & 0.005 & 0.002 & 0.010 & 0.00 & 51 & 0.110 & 0.49 & 0.082 & 44 & 0.440 \\
\hline $10 \mathrm{R}-3,8-12$ & 0.110 & 0.23 & 9.4 & 0.009 & 0.8 & 6.52 & 0.005 & 0.042 & 0.008 & 0.00 & 62 & 0.220 & 0.48 & 0.072 & 47 & 0.270 \\
\hline 11R-1, 102-107 & 0.059 & 0.26 & 11.4 & 0.020 & 0.7 & 3.63 & 0.008 & 0.010 & 0.012 & 0.00 & 42 & 0.210 & 0.62 & 0.096 & 38 & 0.520 \\
\hline $12 \mathrm{R}-1,60-66$ & 0.120 & 0.27 & 8.6 & 0.017 & 1.6 & 2.79 & 0.006 & 0.006 & 0.010 & 0.06 & 36 & 0.130 & 0.54 & 0.081 & 41 & 0.260 \\
\hline $13 \mathrm{R}-2,20-25$ & 0.045 & 0.11 & $\begin{array}{l}0.0 \\
9.9\end{array}$ & 0.002 & 84.5 & 2.55 & 0.004 & 0.004 & 0.008 & 0.00 & 50 & 0.026 & 0.45 & 0.074 & $\begin{array}{l}41 \\
49\end{array}$ & 0.130 \\
\hline $14 \mathrm{R}-3,68-74$ & 0.028 & 0.18 & 9.9 & 0.000 & 0.5 & 2.77 & 0.003 & 0.002 & 0.008 & 0.00 & 39 & 0.068 & 0.36 & 0.067 & 47 & 0.070 \\
\hline $15 \mathrm{R}-1,15-20$ & 0.061 & 0.32 & 9.8 & 0.003 & 0.7 & 3.07 & 0.005 & 0.006 & 0.008 & 0.00 & 44 & 0.088 & 0.43 & 0.073 & 48 & 0.140 \\
\hline $16 R-5,27-32$ & 0.027 & 0.18 & 10.4 & 0.002 & 0.7 & 3.04 & 0.005 & 0.002 & 0.008 & 0.00 & 49 & 0.055 & 0.46 & 0.079 & 40 & 0.070 \\
\hline $17 R-4,75-81$ & 0.036 & 0.23 & 11.1 & 0.003 & 0.6 & 2.81 & 0.005 & 0.008 & 0.009 & 0.00 & 51 & 0.050 & 0.48 & 0.082 & 40 & 0.071 \\
\hline $18 \mathrm{R}-4,15-20$ & 0.043 & 0.26 & 10.4 & 0.012 & 1.6 & 2.32 & 0.006 & 0.002 & 0.010 & 0.00 & 47 & 0.028 & 0.55 & 0.089 & 38 & 0.170 \\
\hline $19 R-2,46-51$ & 0.057 & 0.22 & 10.0 & 0.007 & 1.2 & 1.78 & 0.005 & 0.002 & 0.008 & 0.00 & 51 & 0.011 & 0.41 & 0.074 & 44 & 0.096 \\
\hline $20 \mathrm{R}-2,33-48$ & 0.160 & 0.86 & 10.7 & 0.021 & 1.0 & 2.11 & 0.007 & 0.017 & 0.011 & 0.01 & 49 & 0.072 & 0.55 & 0.096 & 45 & 0.610 \\
\hline $2 \mathrm{IR}-2,74-79$ & 0.043 & 0.14 & 8.7 & 0.000 & 0.6 & 2.77 & 0.003 & 0.004 & 0.006 & 0.00 & 45 & 0.160 & 0.32 & 0.060 & 42 & 0.200 \\
\hline $22 \mathrm{R}-5,37-43$ & 0.049 & 0.23 & 11.1 & 0.003 & 0.9 & 2.75 & 0.005 & 0.005 & 0.009 & 0.00 & 51 & 0.660 & 0.48 & 0.083 & 41 & 0.160 \\
\hline $22 \mathrm{R}-6,18-24$ & 0.080 & 0.31 & 9.3 & 0.002 & 0.9 & 2.76 & 0.003 & 0.006 & 0.006 & 0.00 & 39 & 0.750 & 0.35 & 0.067 & 47 & 0.160 \\
\hline
\end{tabular}

Note: $*=$ X-ray fluorescence, $* *$ = ICP-MS. 
Table 13. Platinum group element (PGE) and gold contents in serpentinized peridotites.

\begin{tabular}{|c|c|c|c|c|c|c|c|c|c|}
\hline $\begin{array}{l}\text { Core, section, } \\
\text { interval }(\mathrm{cm})\end{array}$ & $\begin{array}{c}\text { Os } \\
(\mathrm{ppb})\end{array}$ & $\begin{array}{c}\text { Ir } \\
(\mathrm{ppb})\end{array}$ & $\begin{array}{c}\text { Rul } \\
(\mathrm{ppb})\end{array}$ & $\begin{array}{c}\mathrm{Rh} \\
(\mathrm{ppb})\end{array}$ & $\begin{array}{c}\mathrm{Pt} \\
(\mathrm{ppb})\end{array}$ & $\begin{array}{c}\mathrm{Pd} \\
(\mathrm{ppb})\end{array}$ & $\underset{(\mathrm{ppb})}{\mathrm{Au}}$ & $\frac{\mathrm{Os}+\mathrm{Ir}+\mathrm{Ru}}{\mathrm{Rh}+\mathrm{Pt}+\mathrm{Pd}}$ & $\begin{array}{l}\text { PGE sum } \\
\text { (ppb) }\end{array}$ \\
\hline \multicolumn{10}{|l|}{ 153-920B- } \\
\hline $1 W-3,55-61$ & 2 & 3.9 & 15 & 4.5 & $<5$ & $<3$ & 1.4 & 2.5 & 29.4 \\
\hline $4 R-1,64-68$ & 4 & 4.5 & 15 & 1.1 & $<5$ & 40 & 0.7 & 1.7 & 37.1 \\
\hline $6 \mathrm{R}-3,13-18$ & $<2$ & 2.3 & 15 & 2.4 & 16 & 20 & 2.5 & 0.5 & 56.7 \\
\hline $7 R-2,50-55$ & $<2$ & 2.6 & 15 & 4.2 & $<5$ & $<4$ & 3.0 & 2.3 & 28.3 \\
\hline $10 \mathrm{R}-1,40-45$ & $<2$ & 2.8 & 15 & 1.5 & $<5$ & $<3$ & 1.4 & 3.4 & 24.3 \\
\hline $13 R-3,21-26$ & $<2$ & 3.0 & 15 & 4.4 & $<5$ & $<4$ & 4.5 & 2.1 & 27.9 \\
\hline \multicolumn{10}{|l|}{ 153-920D- } \\
\hline SR-1, 49-55 & 10 & 4.6 & 15 & 8.2 & $<5$ & $<5$ & 1.9 & 2.2 & 42.8 \\
\hline $8 R-2,62-69$ & $<2$ & 2.8 & 15 & 2.3 & $<5$ & $<6$ & 2.8 & 2.4 & 26.6 \\
\hline $11 \mathrm{R}-1,102-107$ & $<2$ & 3.0 & 15 & 4.2 & $<5$ & $<4$ & 1.6 & 2.2 & 27.7 \\
\hline $16 \mathrm{R}-5,27-32$ & $<2$ & 3.8 & 15 & 2.7 & $<5$ & $<5$ & 2.5 & 2.6 & 27.5 \\
\hline $17 R-4,75-81$ & $<2$ & 3.3 & 15 & 3.3 & $<5$ & $<5$ & 3.6 & 2.3 & 27.6 \\
\hline $18 \mathrm{R}-4,15-20$ & 6 & 4.4 & 15 & 6.5 & $<5$ & $<7$ & 2.3 & 2.0 & 37.9 \\
\hline $19 \mathrm{R}-2,46-51$ & $<2$ & 3.2 & 15 & 6.0 & $<5$ & 19 & 4.6 & 0.7 & 46.7 \\
\hline $22 R-5,37-43$ & $<2$ & 3.5 & 15 & 7.0 & $<5$ & $<5$ & 2.9 & 1.6 & 31.5 \\
\hline
\end{tabular}

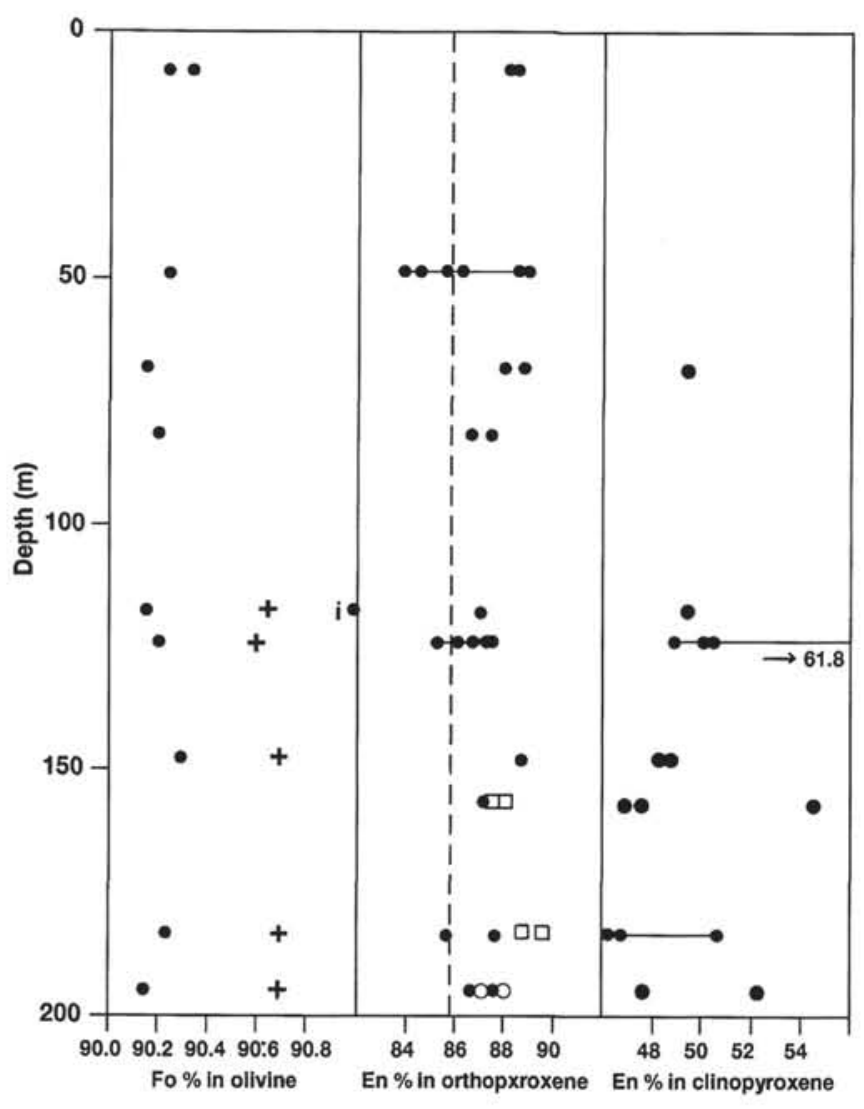

Figure 3. Variation of Fo (molar proportion) in undulatory (probably prekinematic) olivine and En (molar proportion) in undulatory (probably pre- to synkinematic) pyroxenes from peridotites in Hole 920D. Orthopyroxene with En $5.8 \mathrm{~mol} \%$ is classified as pigeonite. Symbols for olivine: dots = undulatory olivine, $\mathrm{i}=$ olivine inclusion in chromite, crosses = olivine associated with chromium-bearing spinel. Symbols for orthopyroxene: dots = porphyroclasts, open circles = undulatory matrix grains, squares = grains in mosaic aggregates. Symbols for clinopyroxene: dots = porphyroclasts. Joined dots represent microprobe profiles in single clasts.

place along the En-Wo join. Again, an increase of the En content is mainly compensated by a decrease in the Wo content. A similar trend was recorded for associated orthopyroxenes and clinopyroxenes in ophiolitic and oceanic peridotite tectonites (e.g., Quick, 1981; Arai and Fuji, 1979; Evans and Girardeau, 1988).

One analysis from the rim of a porphyroclast (number 4 in Table 7) has the composition Wo 31.2, En 61.9, and Fs 6.9, thus deviating markedly from the "normal" porphyroclasts. Analyses from two

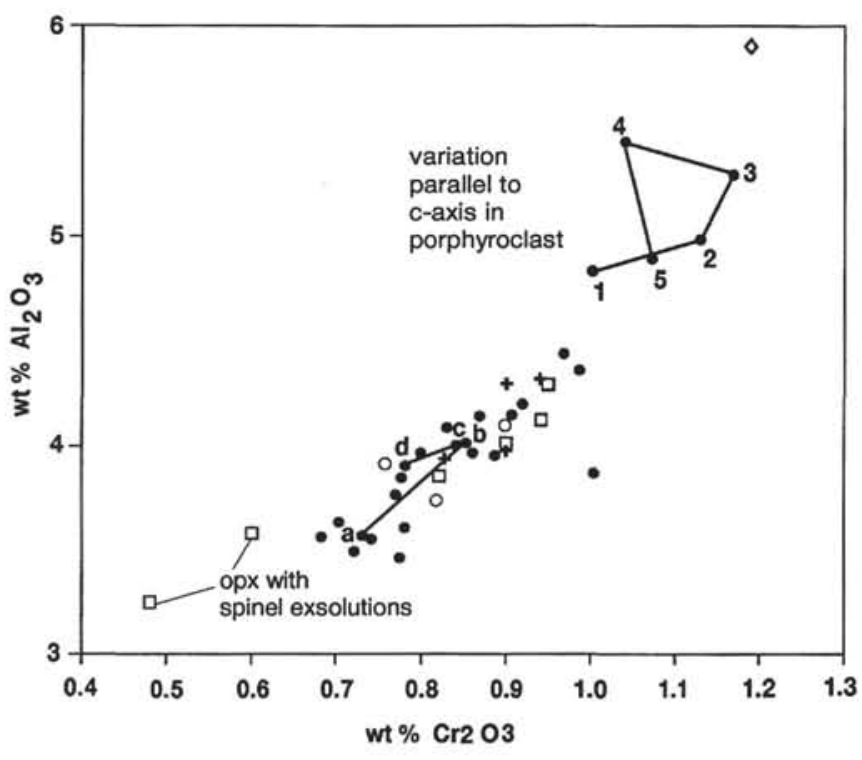

Figure 4. $\mathrm{Al}_{2} \mathrm{O}_{3}$ vs. $\mathrm{Cr}_{2} \mathrm{O}_{3}$ for orthopyroxene (opx) from peridotites, Holes $920 \mathrm{~B}$ and 920D. For symbols, see Figure 3. Diamond = exsolution in clinopyroxene. Joined dots $=$ microprobe profiles in single porphyroclasts.

small deformed and weakly exsolved grains (numbers 1 and 2 in Table 8) show still higher En contents ( 66.3 and $67.5 \mathrm{~mol} \%$ ).

Analyses of exsolved clinopyroxene (morphological variety d) show slightly higher Wo contents than the porphyroclasts and small deformed grains at comparable En and Fs contents (Wo $=48.6-50.1$, $\mathrm{En}=46.1-50.1$, Fs = 3.6-4.3). At different sites and in different habits, exsolved clinopyroxenes within a given orthopyroxene have almost identical compositions despite the chemical heterogeneity of their host. Interstitial clinopyroxenes (morphological variety e) display by far the most wide compositional range (Wo $=40.7-49.3$, En $=46.7-53.8$, Fs $=3.9-5.5$ ).

$\mathrm{Al}_{2} \mathrm{O}_{3}$ contents in the various morphological types of clinopyroxene vary between 3.24 and $5.62 \mathrm{wt} \%$ (Tables 6-10). There is no systematic variation of aluminum with morphological type.

In Figure 5, $\mathrm{TiO}_{2}$ and $\mathrm{Mg} \#$ of the various morphological varieties of orthopyroxenes and clinopyroxenes are compared. The orthopyroxenes have, in general, lower titanium contents and a slightly lower $\mathrm{Mg} \#$ than the clinopyroxenes. The highest $\mathrm{Ti}$ is shown by exsolved clinopyroxene in orthopyroxene porphyroclasts and by interstitial clinopyroxenes. The Ti enrichment in exsolved clinopyroxene supplies evidence of higher initial Ti contents in the orthopyroxene host grain. This is supported by the Ti distribution in a large, scarcely exsolved orthopyroxene porphyroclast (profile numbers 1-5 in Fig. 5). A $\mathrm{TiO}_{2}$ content around $0.1 \mathrm{wt} \%$ can be taken as an initial value, higher than 


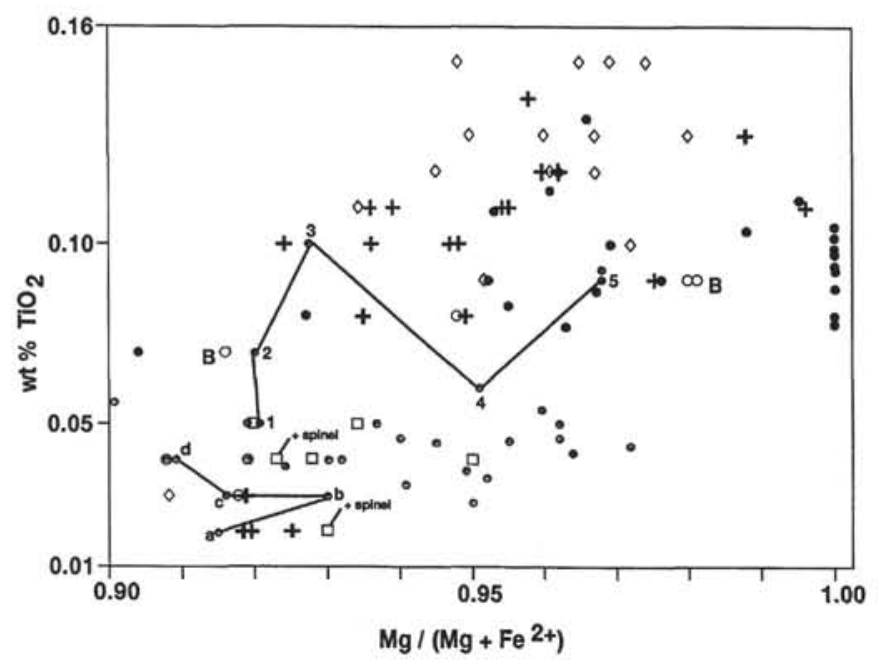

Figure 5. $\mathrm{TiO}_{2}$ vs. $\mathrm{Mg} \#$ for orthopyroxenes and clinopyroxenes from peridotites, Holes 920B and 920D. Symbols for orthopyroxene (lower part of the plot): small open circles $=$ porphyroclasts, large open circles $=$ small undulatory grains, squares = undulatory grains in mosaic aggregates, crosses = undeformed polygonal grains, diamond = exsolution in clinopyroxene, Joined dots = microprobe profiles in two single clasts. Symbols for clinopyroxene (upper part of the plot): dots = porphyroclasts, circles with suffix $\mathrm{B}=$ small deformed grains in samples from Hole 920B, crosses = interstitial undeformed grains, diamonds $=$ exsolutions in orthopyroxene porphyroclasts.

$\mathrm{TiO}_{2}$ contents in strongly exsolved orthopyroxene porphyroclasts $(<0.06 \mathrm{wt} \%)$.

The deformed clinopyroxenes display a wide range of $\mathrm{Al}_{2} \mathrm{O}_{3}$ and $\mathrm{Cr}_{2} \mathrm{O}_{3}$ contents (compare Tables $6-8$ ), but both oxides are usually higher than in deformed orthopyroxenes. In addition, many of our samples show a negative correlation between the $\mathrm{Al}_{2} \mathrm{O}_{3}$ and $\mathrm{Cr}_{2} \mathrm{O}_{3}$ contents of coexisting orthopyroxene and clinopyroxene.

\section{Spinel}

Chromium-bearing spinel is irregularly distributed in the investigated peridotites (modal percentage $0.5 \%-1.5 \%$ ). Depending on the associated silicates, various grain morphologies can be distinguished:

a. Euhedral to subhedral inclusions in olivine and orthopyroxene porphyroclasts.

b. Subhedral and rounded lobate grains (also as "holly leaves") with thornlike extensions between olivine grains (sizes up to $2.5 \mathrm{~mm}$ ).

c. Skeletal and "holly leaf" grains in interstices of small orthopyroxene grains (mosaic grains and neoblasts) and with thornlike extensions; with round olivine inclusions (PI. 2, Fig. 4).

d. Vermiform, amoeboidal, and "holly-leaf" grains in myrmekitic textural relationship with bastitized matrix orthopyroxene (Pl. 1, Fig. 2).

e. Small, homogeneous, more regularly formed and rounded grains in the interstices of orthopyroxene mosaic aggregates (up to $0.05 \mathrm{~mm}$ ).

f. Thin, intergranular films (0.01-mm thickness) between recrystallized and interstitial clinopyroxene grains.

g. Skeletal grains between orthopyroxene clasts.

In general, the spinel grain boundaries are smooth or lobate against olivine, but irregular and hook-shaped against deformed orthopyroxene (except for morphologies a and e).

\section{Chemistry}

Fifteen microprobe analyses from 11 samples are listed in Table 11. The spinels are classified as picotites and in the $\mathrm{Cr} /(\mathrm{Cr}+\mathrm{Al})$ vs. $\mathrm{Fe} /(\mathrm{Fe}+\mathrm{Mg})$ diagram, they plot close to the field of spinels in alpinetype lherzolites. The different morphological spinel varieties appear chemically similar, but they display some variation along the $\mathrm{Mg}-\mathrm{Fe}$ join (Fig. 6).

The two analyses with lowest $\mathrm{Cr} /(\mathrm{Cr}+\mathrm{Al})$ are from vermiform "myrmekitic" spinels at the boundary of slightly deformed granular orthopyroxene (Table 11; analyses 11,12). The orthopyroxenes are characterized by the lowest $\mathrm{Cr}_{2} \mathrm{O}_{3}$ and $\mathrm{Al}_{2} \mathrm{O}_{3}$ contents (Table 4; analyses 4, 5; Fig. 4).

\section{Bulk Chemical Composition}

Rotated factor analysis and correlation matrix were calculated for the elements, which were determined in 34 samples of ultramafic rocks (see Table 12). Figure 7 shows the correlation matrix for these elements, excluding REE, Y, and $\mathrm{Zr}$. The order of the elements in the correlation matrix is derived from the rotated factor analysis, i.e., the groups of elements formed by rotated factor analysis have been used as the succession of elements in the correlation matrix. The correlations with LOI are shown under the correlation matrix.

The second group from the rotated factor analysis, responsible for $22 \%$ of variations in rock composition, contains the elements $\mathrm{Sc}, \mathrm{Al}$, $\mathrm{V}, \mathrm{Si}, \mathrm{Ti}$, and $\mathrm{Cr}$. They are negatively correlated with LOI. Within the next four groups, there is no statistically significant correlation with LOI.

The REE patterns of the investigated ultramafic rocks, normalized on a chondrite basis (Fig. 8) show basically two different patterns. Twenty-three samples show U-shaped patterns and have very low average REE contents. The remaining 11 samples have REE patterns with positive slopes (positive correlation of normalized REE contents with atomic number) and contain higher amounts of REEs than samples with U-shaped pattern.

The most conspicuous feature in REE patterns is the positive europium anomaly occurring in four samples with U-shaped REE pattern and in eight samples with REE patterns having positive slope. There is no significant cerium anomaly in the REE patterns of the investigated ultramafic rocks.

In Figure 9, the statistically significant correlation coefficients between the REEs and other elements are shown. In this figure, different behavior of light REEs (LREEs) in comparison to heavy REEs (HREEs) can be observed. LREEs, especially Eu, are negatively correlated with elements of the second group in rotated factor analysis ( $\mathrm{Sc}, \mathrm{Al}, \mathrm{V}, \mathrm{Si}$, and Ti; see Fig. 7). Unlike the LREEs, the HREEs correlate positively with the above elements. Besides that, $\mathrm{Fe}$ and $\mathrm{Sr}$ have positive correlations to LREEs, and $\mathrm{Mg}$ reveals negative correlations with HREEs.

The correlations between REEs and LOI are all below the level of statistical significance, but we present them in Figure 9 because there is a continuous trend from positive correlations between LOI and LREEs to negative correlations between LOI and HREEs.

Whole-rock contents of platinum group elements were determined for 14 samples of ultramafic rocks. There was no petrographic criterion for selecting these samples, only those samples with enough material remaining after other investigations were analyzed. In Table 13 , only $\mathrm{Ir}, \mathrm{Ru}$, and $\mathrm{Rh}$ were in all samples above the detection limit. Normalized on a chondrite basis, the PGEs concentrate around 0.01 .

The impossibility of determining whether the trends in chondritenormalized PGE patterns decreased or increased compelled us to assess these trends by means of the ratio of the Ir group to the Pt group, i.e., to calculate the ratios of $(\mathrm{Os}+\mathrm{Ir}+\mathrm{Ru}) /(\mathrm{Rh}+\mathrm{Pt}+\mathrm{Pd})$. In cases of elements below the detection limit, we used half of the detection limit as the value (Goldschmidt, 1923-1938). The factors and the sum of all PGEs are included in Table 13. 


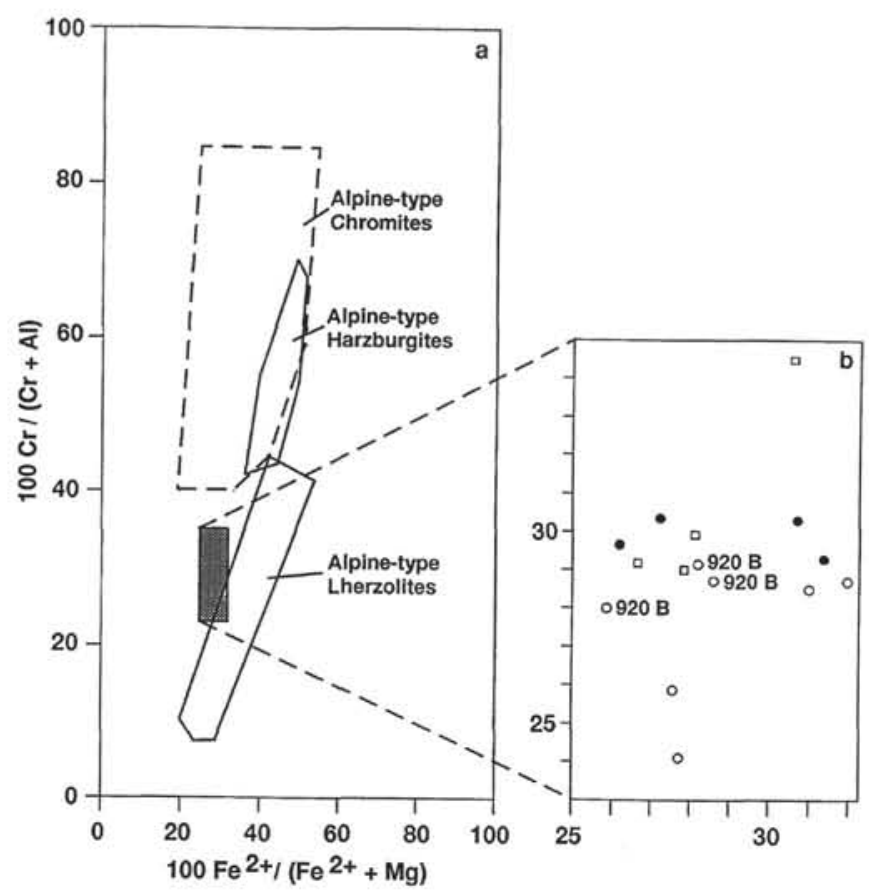

Figure 6. $\mathrm{Cr} /(\mathrm{Cr}+\mathrm{Al})$ vs. $\mathrm{Fe} /(\mathrm{Fe}+\mathrm{Mg})$ for chromium-bearing spinels from peridotites, Holes 920B and 920D. Basic diagram is from Irvine (1967). Field of chromite in alpine-type chromitites is from Burgath and Mohr (1986). Fields of spinels in alpine-type harzburgites and lherzolites are from Roberts (1988). Dots $=$ lobate grains intergrown with olivine, squares $=$ lobate grains in regular intergrowth with bastitized orthopyroxene, circles = myrmekitic spinels (with suffix 920B $=$ from Hole 920B).

The factors $(\mathrm{Os}+\mathrm{Ir}+\mathrm{Ru}) /(\mathrm{Rh}+\mathrm{Pt}+\mathrm{Pd})$ are in most cases higher than 1, oscillating between 1.6 and 3.4. This means that the chondrite-normalized PGE patterns, in which the elements are lined up in order of decreasing melting temperature, as suggested by Naldrett et al. (1979), reveal a negative slope. Only in two samples is the factor $(\mathrm{Os}+\mathrm{Ir}+\mathrm{Ru}) /(\mathrm{Rh}+\mathrm{Pt}+\mathrm{Pd})$ lower than $1(0.5$ and 0.7 , respectively). These samples also contain a higher sum of all PGEs, thus indicating that the low value of the factor is due to enrichment of $\mathrm{Rh}, \mathrm{Pt}$, and $\mathrm{Pd}$ and not due to depletion of $\mathrm{Os}, \mathrm{Ir}$, and $\mathrm{Ru}$. Average values for groups of samples with similar normalized PGE patterns are plotted in Figure 10.

\section{CONCLUSIONS}

The investigated sections from Holes 920B and 920D are strongly serpentinized harzburgites and lherzolites (olivine rich and clinopyroxene poor). Olivine, orthopyroxene, and clinopyroxene are present in a number of morphological varieties. For each mineral, the varieties can be arranged in a chronological order on the basis of textural relationships and the degree of deformation.

The chemical composition of large olivine clasts is comparable to that found in olivines from mantle peridotites in other oceanic areas and from ophiolitic mantle peridotites in mid-ocean-ridge-type ophiolites (Fo around 90, $\mathrm{CaO}=0.05 \mathrm{wt} \%, \mathrm{NiO}=0.4 \mathrm{wt} \%$ ).

The small grains, either undulatory or undeformed, have similar compositions. Thus the textural evolution was obviously not accompanied by noticeable changes in composition.

Of particular interest are the large orthopyroxene clasts and the mosaics of small undulatory grains. Nielsen Pike and Scharzmann (1977) defined the porphyroclasts in peridotites as relicts of a coarser grained primary rock. Mercier and Nicolas (1975) reached a similar conclusion and proposed that porphyroclastic fabrics derived from a former protogranular fabric.

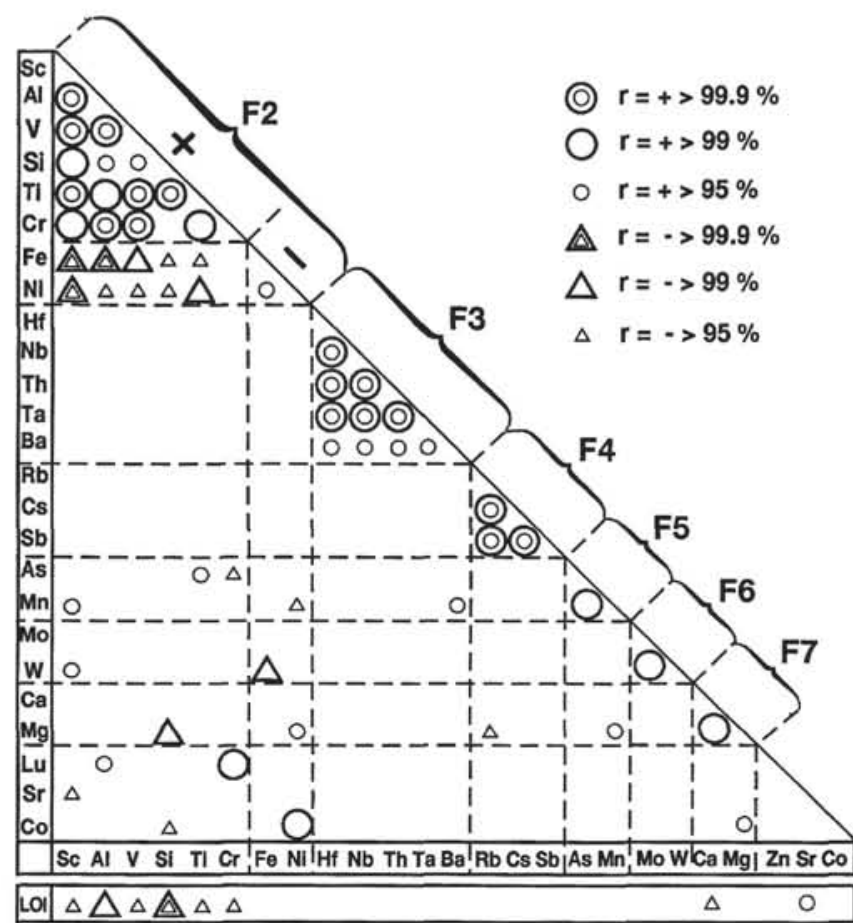

Figure 7. Correlation matrix for all elements determined by bulk analysis of 34 peridotite samples, except REE, Y, and Zr. The order of elements is taken from rotated factor analysis, the factors are marked along the hypotenuse; $r=$ correlation coefficient.

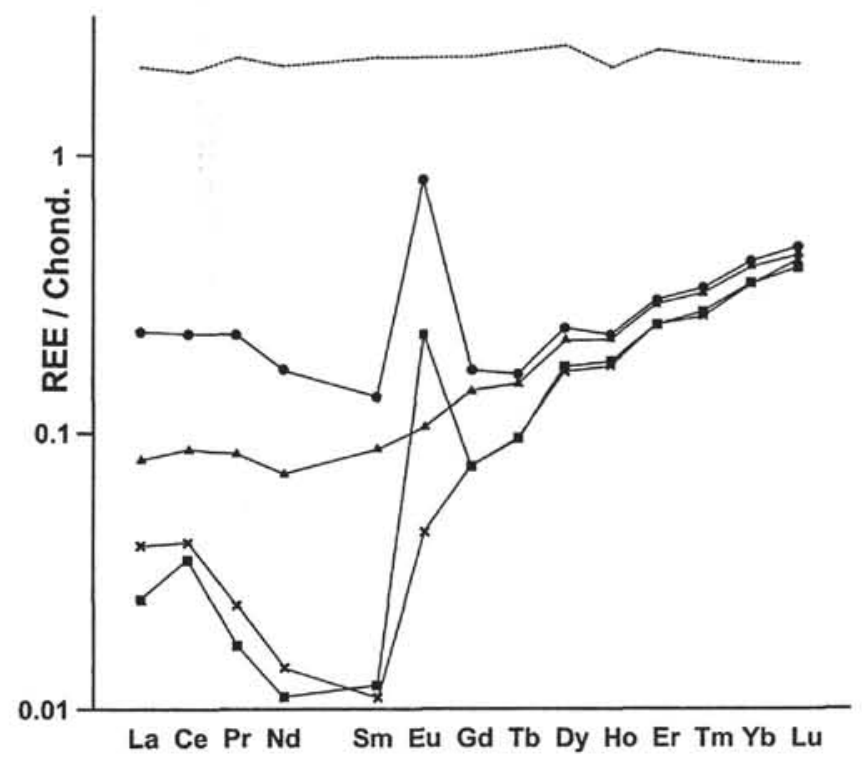

Figure 8. Average REE patterns calculated on a chondrite (Chond.) basis (Wakita et al., 1971). From top to bottom, the averages are as follows: interrupted line $=$ primitive mantle, dots $=$ less-depleted mantle peridotites with positive Eu anomaly (seven samples), triangles $=$ less-depleted mantle peridotites without Eu anomaly (four samples), $\mathrm{x}=$ depleted mantle peridotites without Eu-anomaly (19 samples), squares $=$ depleted mantle peridotites with positive Eu-anomaly (four samples).

Several orthopyroxene porphyroclasts in our samples have high $\mathrm{Ca}$ contents $(\mathrm{CaO}>2.7 \mathrm{wt} \%)$ despite extensive clinopyroxene exsolution.

Generally, the large clasts are characterized by elevated $\mathrm{Ni}$ and $\mathrm{Cr}$ contents $\left(\mathrm{NiO}=0.07-0.14 \mathrm{wt} \% ; \mathrm{Cr}_{2} \mathrm{O}_{3}=0.6-1.2 \mathrm{wt} \%\right)$, when compared with smaller orthopyroxenes. The latter (individual small 


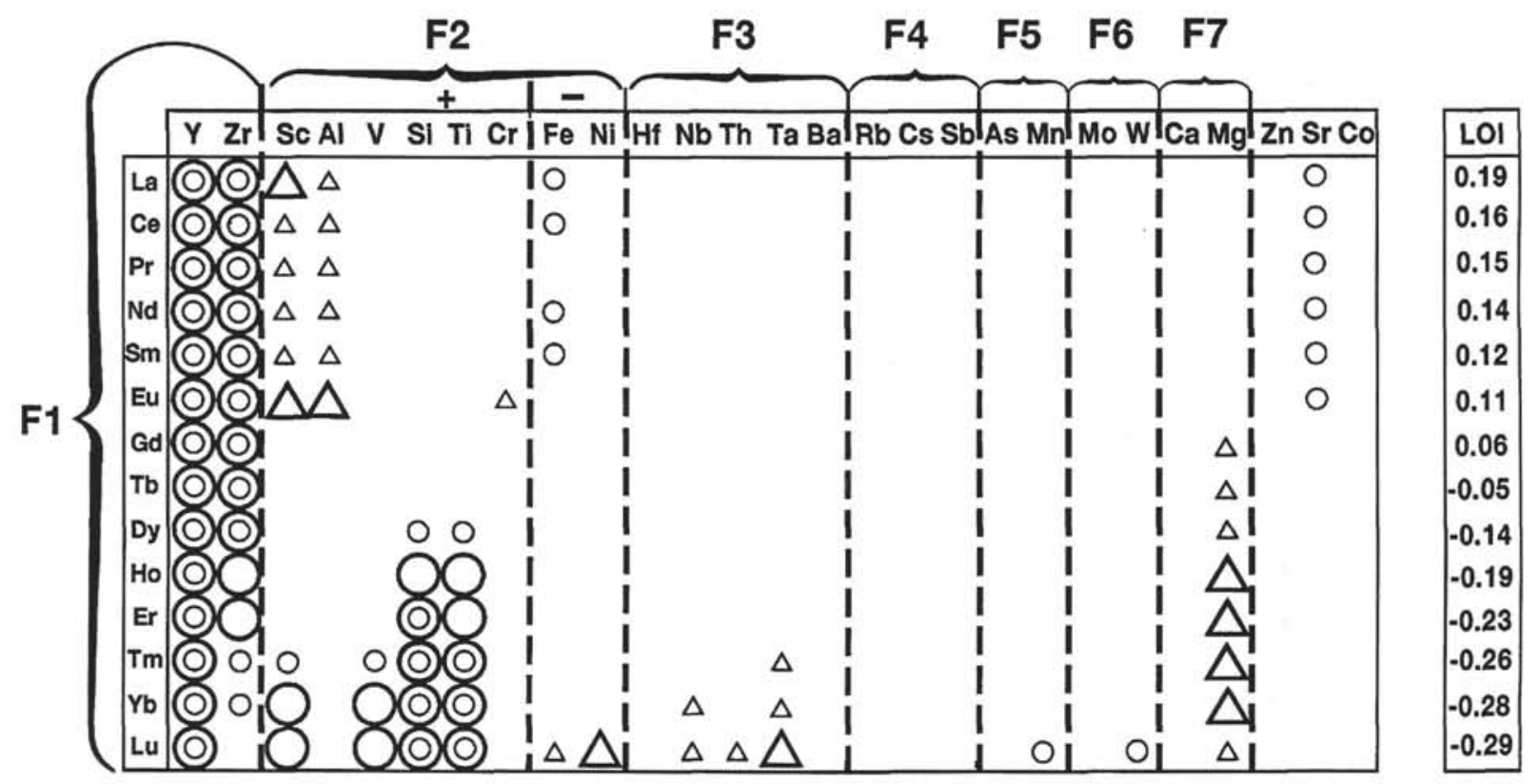

Figure 9. Correlations between REE and other elements analyzed for 34 peridotite samples from Holes $920 \mathrm{~B}$ and $920 \mathrm{D}$. The symbols are the same as in Figure 7. The order of elements is taken from rotated factor analysis, and the factors are marked. Only statistically significant correlations are plotted, except for correlations with LOI, which are all below the level of significance.

grains and anhedral grains in mosaic aggregates) have, on average, lower Wo proportions, $\mathrm{Mg}$ numbers, $\mathrm{Cr}, \mathrm{Ni}$, and $\mathrm{Ti}$ contents. Textural evidence for the formation of the mosaic aggregates via mechanical dismembering of large clasts (with redistribution of the above elements into exsolved clinopyroxene) is the absence of olivine and the general appearance of interstitial clinopyroxene in the mosaics. This clinopyroxene probably developed from enlargement of exsolutions (see below). In our samples, evidence for a possible origination of the porphyroclasts or mosaic aggregates from orthopyroxene layers or dikes is not present. The morphologies of clinopyroxene and their chronological order are similar to those of orthopyroxene, including deformed clasts, exsolution lamellae in orthopyroxene clasts, and interstitial grains. The origin of interstitial clinopyroxene is of particular interest. Clinopyroxenes of this type (not deformed, often twinned, and not exsolved) were described from mantle peridotites in ophiolites (e.g., Evarts, 1977; Sinton, 1977; George, 1978; Quick, 1981 ) and from numerous mid-ocean ridge localities (e.g., Boudier, 1979; Cannat et al., 1990; Juteau et al., 1990). These and other authors (e.g., Nicolas et al., 1980; Boudier and Coleman, 1981; Nicolas, 1986 ) attribute the formation of clinopyroxenes of this type to crystallization from an interstitial melt ("magmatic impregnation" after Nicolas et al., 1980). In our samples, most orthopyroxene porphyroclasts are characterized by extensive clinopyroxene exsolution and near kink-band traces, these exsolutions display a continuous textural gradation to and optical continuity with larger clinopyroxene plates. The plates fill the space between opening kink bands and proceed to the interstices between orthopyroxenes (e.g., in mosaic arrangements). This mechanism of formation of interstitial grains from exsolved clinopyroxene in orthopyroxene has already been envisaged by several authors (Frey and Green, 1974; Mercier and Nicolas, 1975; Hamlyn and Bonatti, 1981; Komor et al., 1990). Porphyroclastic, exsolved, and interstitial clinopyroxenes in our samples, however, have similar compositions. Nevertheless, the clasts have, on average, lower $\mathrm{Na}_{2} \mathrm{O}$ contents $(0.01-0.08 \mathrm{wt} \%$; mean of 18 samples $=$ $0.058)$ than those of the exsolutions $(0.06-0.12 \mathrm{wt} \%$; mean of 14 analyses $=0.085)$ and the interstitial grains $(0.05-0.10 \mathrm{wt} \%$; mean of 16 analyses $=0.082$ ). In addition, $\mathrm{TiO}_{2}$ in the deformed grains is mostly lower than $0.1 \mathrm{wt} \%$ and is between 0.11 and $0.15 \mathrm{wt} \%$ in the exsolutions and interstitial grains. The $\mathrm{Cr}_{2} \mathrm{O}_{3}$ contents in exsolved

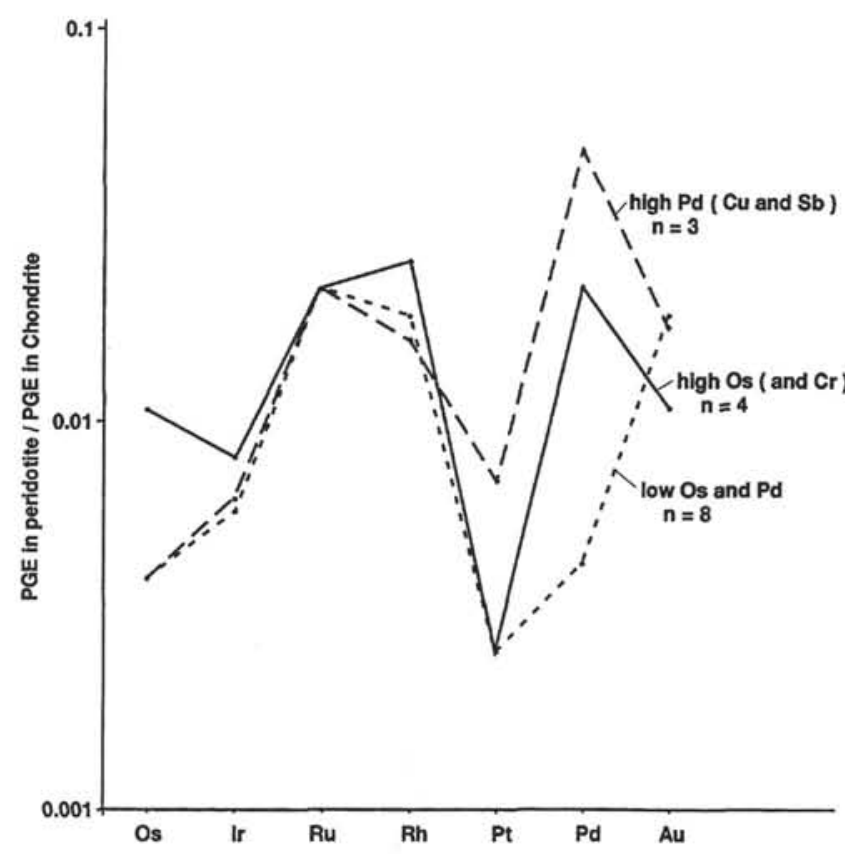

Figure 10. Chondrite-normalized PGE patterns in peridotites from Site 920. Average values are shown for four samples with Os enrichment, three samples with Pd enrichment, and the seven remaining samples. Normalization on a chondrite basis from Naldrett and Duke (1980).

and interstitial clinopyroxenes (mean $=1.37 \mathrm{wt} \%$ for exsolutions and $1.23 \mathrm{wt} \%$ for interstitial grains) are also somewhat higher than in the deformed clinopyroxene varieties.

The most striking habits of spinel are interstitial, subhedral, and lobate grains in olivine arrangements, interstitial irregular and "holly leaf" grains in orthopyroxene-dominated matrix, and "symplectic" intergrowth with orthopyroxene. Chemically, the interstitial grains in spinel-olivine and spinel-orthopyroxene associations are quite similar. We think, therefore, that these spinels have inherited their Al-rich 
character and have adapted their morphology during high-temperature deformation and grain-boundary migration along with the associated silicates.

For the formation of symplectic spinel, an exsolution from enstatite or pigeonite (Menzies, 1975; Mercier and Nicolas, 1975) or a subsolidus reaction between adjacent phases (Leblanc et al., 1980; Lehmann, 1983) have been proposed. In our samples, analyses of orthopyroxene in "symplectic" intergrowth with spinel show the lowest $\mathrm{CaO}, \mathrm{Cr}_{2} \mathrm{O}_{3}$, and $\mathrm{Al}_{2} \mathrm{O}_{3}$ contents of all investigated orthopyroxenes. We think that in this case the spinel resulted from a subsolidus reaction between orthopyroxene and adjacent olivine.

With the information obtained from the statistical evaluation of bulk chemical composition, the chondrite-normalized REE patterns are the most powerful tools to decide how far the investigated part of the upper mantle was modified by partial mantle melting. The Ushaped REE pattern in 23 of the 34 investigated mantle rocks was interpreted by Frey (1984) as typical for depleted mantle with metasomatic enrichment of LREEs. The remaining 11 samples with higher average amounts of REEs and with positive slope are interpreted as typical for less depleted mantle rocks.

In Figure 2, these two different REE patterns, interpreted as strongly and less depleted mantle, have been plotted vs. depth in Holes 920B and 920D. Both holes contain one zone with less depleted mantle material at a depth of less than 50 mbsf (documented by one sample in each hole) and a second zone at a depth of less than 100 mbsf (documented by 3 and 2 samples, respectively). Two additional zones of less depleted mantle occur at the top (one sample) and at a depth between 150 and 200 mbsf ( 3 samples) in Hole 920D. Because of limited sampling, this downhole distribution is probably not significant. We can only conclude that mantle inhomogeneities occur in the two investigated holes, i.e., the zones of strongly depleted mantle alternate with zones of not so strongly depleted mantle.

The negative correlations of $\mathrm{Sc}, \mathrm{Al}, \mathrm{V}, \mathrm{Si}, \mathrm{Ti}$, and $\mathrm{Cr}$ with $\mathrm{LOI}$ (the main indicator of alteration), shown in Figure 7, suggest that this group of elements is representative of the primary composition of ultramafic rocks, unaffected by alteration. The lack of statistically significant correlations, positive or negative, between most other elements and LOI, shown in Figure 7, is interpreted as an indication of some mobility of these elements during alteration or for an initial patchy distribution of these elements in the upper mantle sequence. The groups of elements that are formed by rotated factor analyses can represent groups of elements incorporated in the same secondary mineral or groups of elements that have a similar behavior during alteration processes. The less mobile, high-field-strength elements (HFSEs) are concentrated in the first three groups, whereas the more mobile, large-ion-lithophile elements (LIL) dominate in later groups.

The positive correlation of $\mathrm{Sc}, \mathrm{Al}, \mathrm{V}, \mathrm{Si}$, and $\mathrm{Ti}$ with the HREEs and their negative correlation with the LREEs (Fig. 8) indicate that HREEs, along with $\mathrm{Sc}, \mathrm{Al}, \mathrm{V}, \mathrm{Si}$, and $\mathrm{Ti}$, are indicative of primary rock composition. The LREEs, however, cannot be considered of low mobility during rock alteration. Positive correlations of $\mathrm{Fe}$ and $\mathrm{Sr}$ with LREEs and negative correlations of $\mathrm{Mg}$ with HREEs can be interpreted in a same way; with mobility of these three elements during alteration.

The conspicuous positive Eu anomaly occurring in 12 of 34 analyzed samples cannot be related to plagioclase, as this mineral is absent from the investigated peridotites (after own unpublished results, plagioclase-rich melts, in general, reveal a much lower positive Eu anomaly). High temperature and low oxygen fugacity can provide conditions to reduce part of $\mathrm{Eu}^{3+}$ to $\mathrm{Eu}^{2+}$. Because of this change of ionic potential, the reduced Eu falls from the group of HFSEs to the group of LIL elements, and therefore becomes more mobile. Sun and Nesbitt (1978) also describe the development of positive Eu anomalies in ultramafic rocks and explain them as consequence of alteration.

The four samples with elevated Os contents (Fig. 10) also contain a higher amount of Cr-bearing spinel than the other analyzed sam- ples. Pd enrichment in three samples is supposed to be due to precipitation of hydrothermal sulfides, and can consequently be interpreted as an indicator of alteration. The corresponding samples contain abundant sulfides, and $\mathrm{Cu}$ and $\mathrm{Sb}$, which are commonly bond to sulfide minerals.

\section{REFERENCES}

Arai, S., and Fujii, T., 1979. Petrology of ultramafic rocks from Site 395. In Melson, W.G., Rabinowitz, P.D., et al., Init. Repts. DSDP, 45: Washington (U.S. Govt. Printing Office), 587-594.

Augé, T., 1982. Étude minéralogique et pétrographique des roches basiques et ultrabasiques du complexe ophiolitique du Noral, Oman [These]. Univ. D'Orleans.

Boudier, F., 1979. Microstructural study of three peridotite samples drilled at the western margin of the Mid-Atlantic Ridge. In Melson, W.G., Rabinowitz, P.D., et al., Init. Repts. DSDP, 45: Washington (U.S. Govt. Printing Office), 603-608.

Boudier, F., and Coleman, R.G., 1981. Cross section through the peridotite in the Samail ophiolite, southeastern Oman Mountains. J. Geophys. Res., $86: 2573-2592$.

Burgath, K.P., and Mohr, M., 1986. Chromitites and platinum group minerals in the Meratus-Bobaris Ophiolite Zone, Southeast Borneo. In Gallagher, M.J., Ixer, R.A., Neary, C.R., Prichard, H.M. (Eds.), Metallogeny of Basic and Ultrabasic Rocks. Inst. Min. Metall., London, 339-349.

Burgath, K.P., and Weiser, T., 1980. Primary textures and genesis of greek podiform chromite deposits. In Panayiotou, A. (Ed.), Ophiolites. Geol. Surv. Dept. Cyprus, 675-690.

Cannat, M., Bideau, D., and Hébert, R., 1990. Plastic deformation and magmatic impregnation in serpentinized ultramafic rocks from the Garrett transform fault (East Pacific Rise). Earth Planet. Sci. Lett., 101:216-232.

Cannat, M., and ODP Leg 153 Shipboard Scientific Party, 1995. Probing the foundation of the Mid-Atlantic Ridge. Eos, 76:129-133.

Coleman, R.G., 1977. Ophiolites: Ancient Oceanic Lithosphere: New York (Springer-Verlag).

Dick, H.J.B., Fisher, R.L., and Bryan, W.B., 1984. Mineralogic variability of the uppermost mantle along mid-ocean ridges. Earth Planet. Sci. Lett., 69:88-106.

Evans, C.A., and Girardeau, J., 1988. Galicia Margin peridotites: undepleted abyssal peridotites from the North Atlantic. In Boillot, G., Winterer, E.L., et al., Proc. ODP, Sci. Results, 103: College Station, TX (Ocean Drilling Program), 195-207.

Evarts, R.C., 1977. The geology and petrology of the Del Puerto ophiolite, Diablo Range, Central California Coast Ranges. In Coleman, R.G., and Irwin, W.P. (Eds.), North American Ophiolites. Oregon Dept. Geol. Miner. Ind. Bull., 95:121-140.

Frey, F.A., 1984. Rare earth element abundances in upper mantle rocks. In Henderson, P. (Ed.), Rare Earth Element Geochemistry: Amsterdam (Elsevier), 153-203.

Frey, F.A., and Green, D.H., 1974. The mineralogy, geochemistry and origin of Lherzolitic inclusions in Victorian basanites. Geochim. Cosmochim. Acta., 38:1023-1059.

George, R.P., 1978. Structural petrology of the Olympus ultramafic complex in the Troodos ophiolite, Cyprus. Geol. Soc. Am. Bull., 89:845-865.

Goldschmidt, V.M., 1923-1938. Geochemische Verteilungsgesetze (I-IX). Akad. Wiss. Oslo.

Gueguen, Y., and Nicolas, A., 1980. Deformation of mantle rocks. Ann. Rev. Earth Planet. Sci., 8:119-144.

Hamlyn, P.R., and Bonatti, E., 1981. Petrology of mantle derived ultramafics from the Owen fracture zone, northwest Indian Ocean: implications for the nature of the oceanic upper mantle. Earth Planet. Sci. Lett., 48:965979.

Irvine, T.N., 1967. Chromium spinel as a petrogenetic indicator, Part 2: petrological applications. Can. J. Earth Sci., 4:71-103.

Juteau, T., Berger, E., and Cannat, M., 1990. Serpentinized, residual mantle peridotites from the M.A.R. median valley, ODP Hole $670 \mathrm{~A}\left(21^{\circ} 10 \mathrm{~N}\right.$, $45^{\circ} 02^{\prime} \mathrm{W}$, Leg 109): primary mineralogy and geothermometry. In Detrick, R., Honnorez, J., Bryan, W.B., Juteau, T., et al., Proc. ODP, Sci. Results, 106/109: College Station, TX (Ocean Drilling Program), 27-45.

Karson, J.A., Thompson, G., Humphris, S.E., Edmond, J.M., Bryan, W.B., Brown, J.R., Winters, A.T., Pockalny, R.A., Casey, J.F., Campbell, A.C., Klinkhammer, G., Palmer, M.R., Kinzler, R.J., and Sulanowska, M.M., 1987. Along-axis variations in seafloor spreading in the MARK area. Nature, 328:681-685. 
Komor, S.C., Grove, T.L., and Hébert, R., 1990. Abyssal peridotites from ODP Hole $670 \mathrm{~A}\left(21^{\circ} 10 \mathrm{~N}, 45^{\circ} 02 \mathrm{~W}\right)$ : residues of mantle melting exposed by non-constructive axial divergence. In Detrick, R., Honnorez, J., Bryan, W.B., Juteau, T., et al., Proc. ODP, Sci. Results, 106/109: College Station, TX (Ocean Drilling Program), 85-101.

Leblanc, M., Dupuy, C., Cassard, D., Moutte, J., Nicolas, A., Prinzhoffer, A., Rabinovitch, M., and Routhier, P., 1980. Essai sur la genèse des corps podiformes de chromitite dans les péridotites ophiolithiques: étude des chromites de Nouvelle-Caledonie et comparaison avec celles du Mediterranée orientale. In Panayiotou, A. (Ed.), Ophiolites. Geol. Surv. Dept. Cyprus, 691-701.

Lehmann, J., 1983. Diffusion between olivine and spinel: application to geothermometry. Earth Planet. Sci. Lett., 64:123-138.

Menzies, M., 1975. Spinel compositional variation in the crustal and mantle lithologies of the Othris ophiolite. Contrib. Mineral. Petrol., 51:303-309.

Mercier, J.-C.C., and Nicolas, A., 1975. Textures and fabrics of upper mantle peridotites as illustrated by xenoliths from basalts. J. Petrol., 16:454496.

Mével, C., Cannat, M., Gente, P., Marion, E., Auzende, J.-M., and Karson, J.A., 1991. Emplacement of deep crustal and mantle rocks on the west median valley wall of the MARK area (MAR $23^{\circ} \mathrm{N}$ ). Tectonophysics, 190:31-53.

Naldrett, A.J., and Duke, J.M., 1980. Platinum metals in magmatic sulfide ores. Science, 208:1417-1424.

Naldrett, A.J., Hoffman, E.L., Green, A.H., Chou, C.-L., Naldrett, S.R., and Alcock, R.A., 1979. The composition of Ni-sulphide ores, with particular reference to their content of PGE and Au. Can. Mineral., 17:403-415.

Nicolas, A., 1986. Structure and petrology of peridotites: clues to their geodynamic environment. Rev. Geophys., 24:875-895.

Nicolas, A., Bouchez, J.L., Boudier, F., and Mercier, J.C., 1971. Textures, structures and fabrics due to solid state flow in some European lherzolites. Tectonophysics, 12:55-86.
Nicolas, A., Boudier, F., and Bouchez, J.L., 1980. Interpretation of peridotite structures from ophiolitic and oceanic environments. Am. J. Sci., 280:192-210.

Nicolas, A., and Poirier, J.-P., 1976. Crystalline Plasticity and Solid State Flow in Metamorphic Rocks: New York (Wiley).

Nielsen Pike, J.E., and Scharzman, E.C., 1977. Classification of textures in ultramafic xenoliths. J. Geol., 85:49-61.

Orberger, B., Lorand, J.P., Girardeau, J., Mercier, J.C.C., and Pitragool, S., 1995. Petrogenesis of ultramafic rocks and associated chromitites in the Nan Ultaradit ophiolite, Northern Thailand. Lithos, 35:153-182.

Quick, J.E., 1981. Petrology and petrogenesis of the Trinity Peridotite, an upper mantle diapir in the eastern Klamath Mountains, northern California. J. Geophys. Res., 86:11837-11863.

Roberts, S., 1988. Ophiolitic chromitite formation: a marginal basin phenomenon? Econ. Geol., 83:1034-1036.

Sinton, J., 1977. Equilibration history of the basal alpine-type peridotite, Red Mountain, New Zealand. J. Petrol., 18:216-246.

Sun, S.S., and Nesbitt, R.W., 1978. Petrogenesis of Archean ultrabasic and basic volcanics: evidence from rare earth elements. Contrib. Mineral. Petrol., 65:301-325.

Wakita, H., Rey, P., and Schmitt, R.A., 1971. Abundances of the 14 rareearth elements and 12 other trace-elements in Apollo 12 samples: five igneous and one breccia rocks and four soils. Proc. 2nd Lunar Sci. Conf., 1319-1329.

Date of initial receipt: 8 August 1995

Date of acceptance: 26 February 1996

Ms 153SR-032 
1

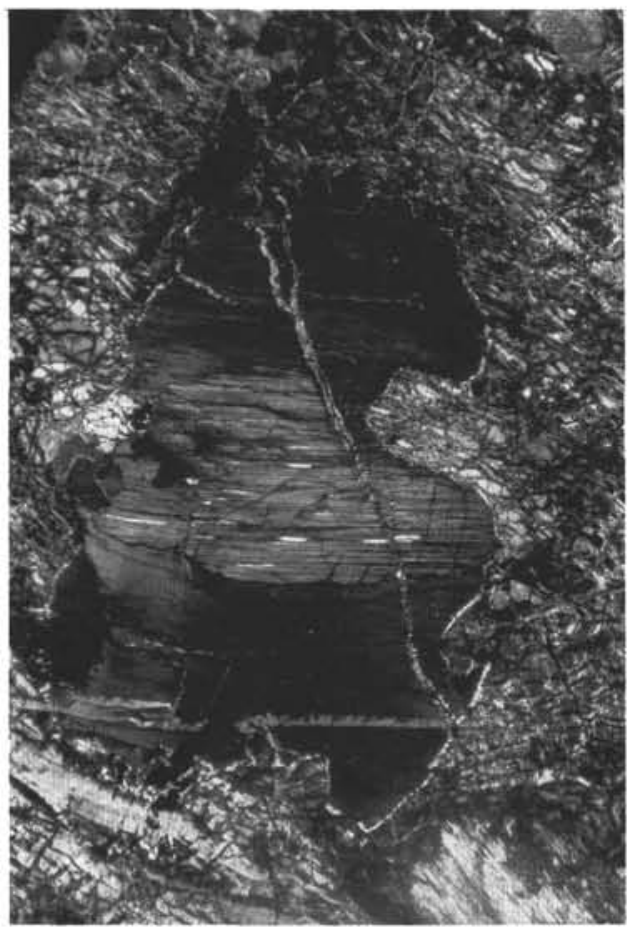

3

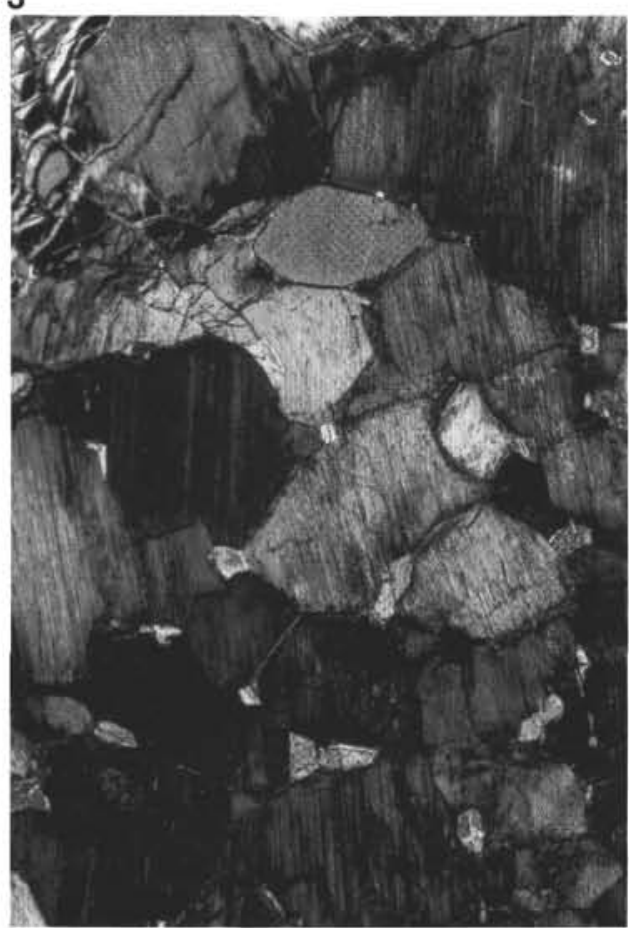

2

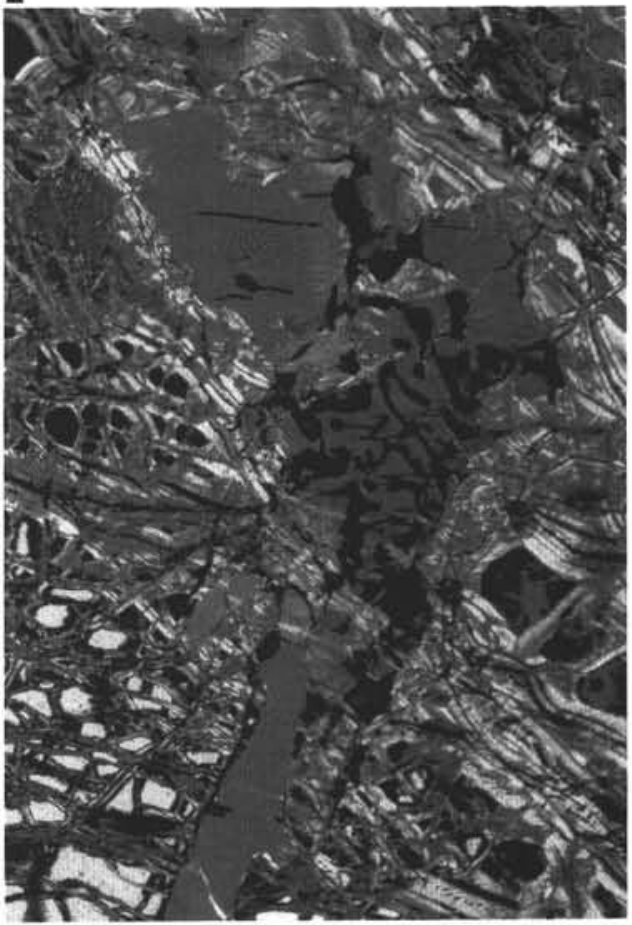

4

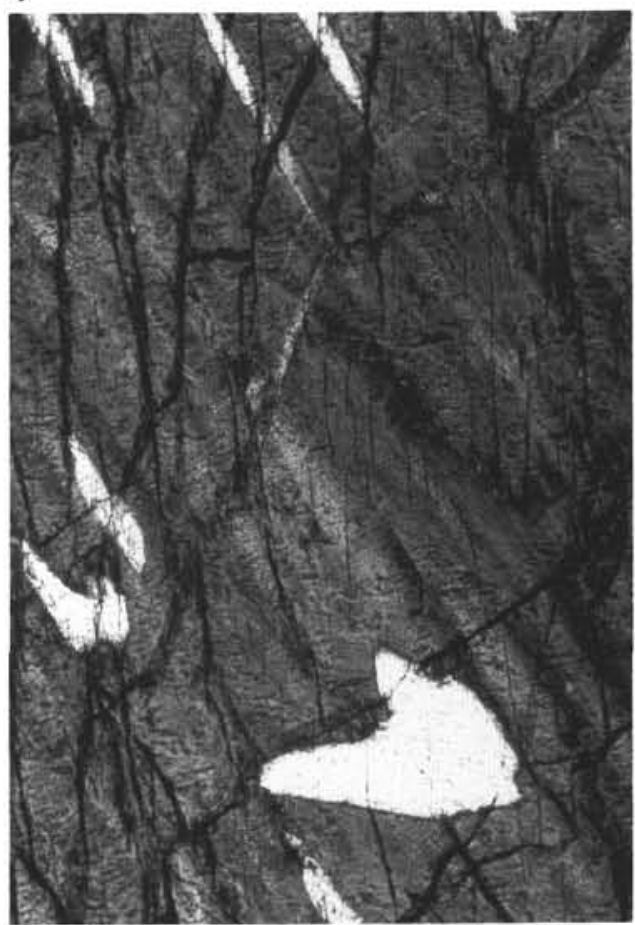

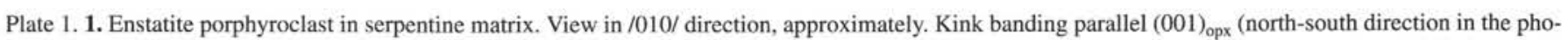
tograph, opx = orthopyroxene). Lamellar and spindle-shaped clinopyroxene exsolutions parallel to $/ 100 /$ opx (horizontal direction). Trace of rock foliation parallel to $/ 001 /_{\text {opx }}$ (horizontal direction). Embayments are filled with small undeformed olivine and orthopyroxene neoblasts). Crossed nicols, width of photograph is $4.9 \mathrm{~mm}$. Serpentinized harzburgite, Sample 153-920D-18R-4, 15-20 cm. 2. "Myrmekitic" intergrowth of bastitized matrix orthopyroxene (gray homogeneous areas) and chromium-bearing spinel. Matrix is mesh serpentine. Crossed nicols, width of photograph is $2.4 \mathrm{~mm}$. Serpentinized harzburgite, Sample 153-920D$22 \mathrm{R}-5,37-43 \mathrm{~cm}$. 3. Mosaic of orthopyroxene grains (striped) with interstitial clinopyroxene (small light grains, as in the lower part of the photograph). These mosaics have probably developed via disintegration of orthopyroxene porphyroclasts. Crossed nicols, width of photograph is $2.4 \mathrm{~mm}$. Serpentinized harzburgite, Sample 153-920D-22R-5, 37-43 cm. 4. Lamellar and platy clinopyroxene exsolutions oblique to /001/ in orthopyroxene host. Largest clinopyroxene plate is twinned (trace of twinning plane is marked). $/ 001 /$ opx is displayed by vertical cleavage orientation. Parallel orientation of /001/ axes in orthopyroxene host and twinned clinopyroxene exsolution. Crossed nicols, width of photograph is $0.6 \mathrm{~mm}$. Serpentinized harzburgite, Sample 153-920D-16R-5, 27-32 cm. 
1

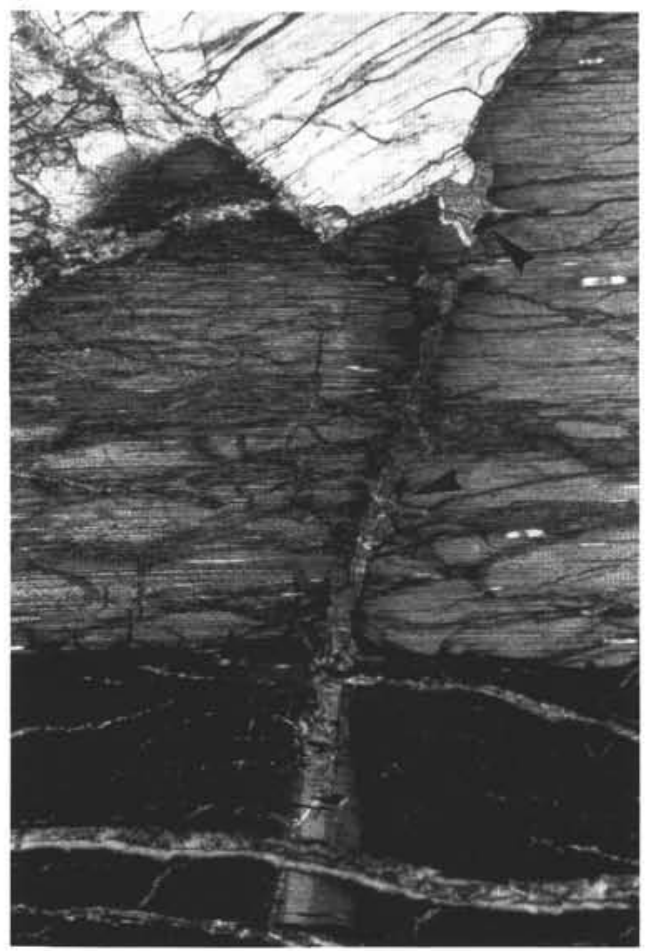

3

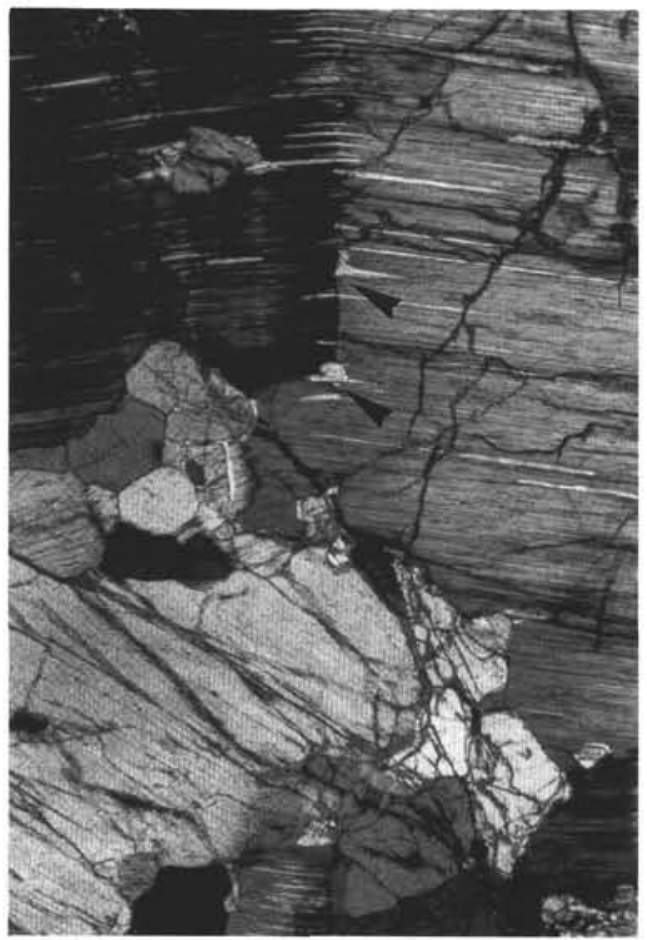

2

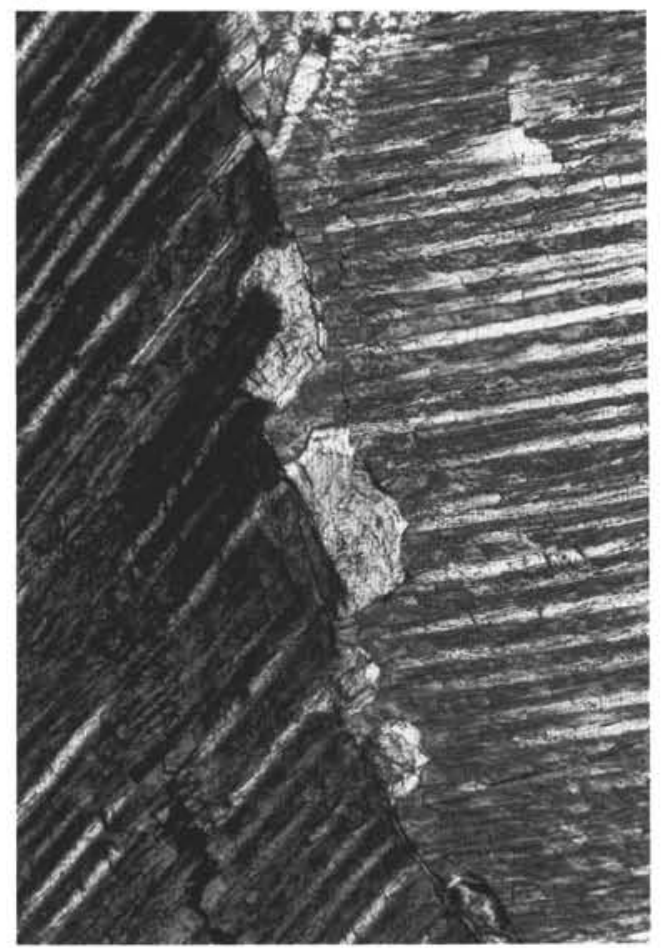

4

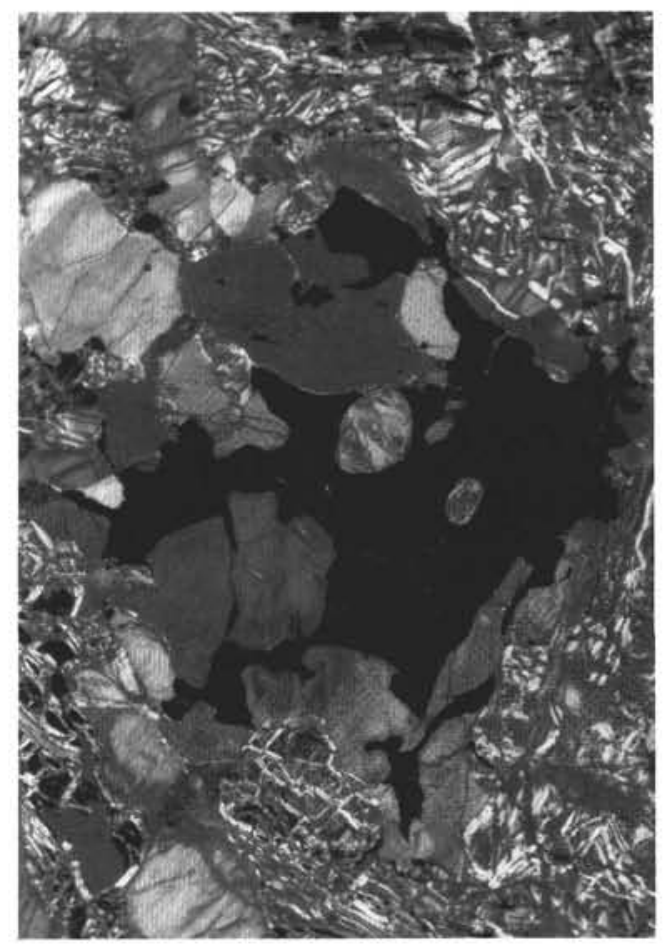

Plate 2. 1. Orthopyroxene porphyroclast with diopside exsolutions (lamellae and short horizontal rods) parallel to /100/opx. The lamellae and rods merge into a vertical ribbon and larger plates of clinopyroxene, which fill the void of an opening kink band (e.g., in the central part of the photograph; see arrows). Crossed nicols, width of photograph is $2.4 \mathrm{~mm}$. Serpentinized harzburgite, Sample 153-920D-18R-4, 15-20 cm. 2. Growth of undeformed orthopyroxene (light gray, center of the photograph) along the contact of two clinopyroxene porphyroclasts. The orthopyroxene probably formed via integration of exsolution lamellae (white lamellae in clinopyroxene). Crossed nicols, width of photograph is $0.6 \mathrm{~mm}$. Serpentinized harzburgite, Sample 153-920B-12R-1, 96-100 cm. 3. Formation of larger clinopyroxene plates (arrows) from exsolution lamellae (white) along vertical kink band in orthopyroxene. Left of center and lower right corner: formation of small polygonal orthopyroxene and clinopyroxene neoclasts on the contact between orthopyroxene clasts and kink band. Crossed nicols, width of photograph is $2.4 \mathrm{~mm}$. Serpentinized harzburgite, Sample 153-920B-13R-3,21-26 cm. 4. Intergrowth of skeletal and lobate spinel "holly leaf" grains with bastitized orthopyroxene grains (undulatory mosaic grains and neoblasts). Round inclusions in spinel are serpentinized olivine. Crossed nicols, width of photograph is $2.4 \mathrm{~mm}$. Serpentinized harzburgite, Sample $153-920 \mathrm{D}-16 \mathrm{R}-5,27-32 \mathrm{~cm}$. 